OPEN ACCESS

Edited by:

Kalijn Fredrike Bol,

University of Copenhagen, Denmark

Reviewed by:

loana Cosgarea,

Newcastle University, United Kingdom

Shahab Babakoohi,

Medical College of Wisconsin,

United States

*Correspondence:

Ivo Abraham

abraham@pharmacy.arizona.edu

Specialty section:

This article was submitted to

Skin Cancer

a section of the journal

Frontiers in Oncology

Received: 12 November 2019 Accepted: 17 January 2020

Published: 11 February 2020

Citation:

Almutairi AR, McBride A, Slack M,

Erstad BL and Abraham I (2020)

Potential Immune-Related Adverse

Events Associated With Monotherapy

and Combination Therapy of

Ipilimumab, Nivolumab, and

Pembrolizumab for Advanced

Melanoma: A Systematic Review and

Meta-Analysis. Front. Oncol. 10:91.

doi: 10.3389/fonc. 2020.00091
Potential Immune-Related Adverse Events Associated With Monotherapy and Combination Therapy of Ipilimumab, Nivolumab, and Pembrolizumab for Advanced

\section{Melanoma: A Systematic Review and Meta-Analysis}

\author{
Abdulaali R. Almutairi ${ }^{1,2}$, Ali McBride ${ }^{2,3}$, Marion Slack $^{2}$, Brian L. Erstad ${ }^{2}$ and \\ Ivo Abraham ${ }^{1,2,3 *}$
}

\begin{abstract}
${ }^{1}$ Center for Health Outcomes and PharmacoEconomic Research, College of Pharmacy, University of Arizona, Tucson, AZ, United States, ${ }^{2}$ Department of Pharmacy Practice and Science, College of Pharmacy, University of Arizona, Tucson, AZ, United States, ${ }^{3}$ University of Arizona Cancer Center, Tucson, AZ, United States
\end{abstract}

Background: The use of ipilimumab, nivolumab, and pembrolizumab as monotherapies or in combination has transformed the management of advanced melanoma even though these drugs are associated with a new profile of immune-related adverse events (irAEs). The incidence of irAEs from clinical trials of these agents is an important factor for clinicians when treating patients with advanced melanoma. In the current study, we aimed to profile the incidence of potential irAEs of these agents when used as monotherapy and as combination therapy.

Methods: We searched the Medline, Embase, and Cochrane databases; clinicaltrials.gov; and websites of regulatory agencies in the USA, Europe, Australia, and Japan for phase 1-3 trials of ipilimumab, nivolumab, and pembrolizumab for advanced melanoma. Random effect meta-analysis was utilized to profile the incidence of potential irAEs.

Results: A total of 58 reports of 35 trials including 6,331 patients with advanced melanoma and reporting irAE data were included in the meta-analyses. We found higher incidences of potential irAEs in combination therapies vs. monotherapies for most of the types of irAEs. Among the monotherapies, ipilimumab users had the most frequent incidence of potential irAEs related to the gastrointestinal system (diarrhea, 29\%; and colitis, 8\%) and skin (rash, 31\%; pruritus, 27\%; and dermatitis, 10\%), with hypophysitis in $4 \%$ of the patients. The most frequent potential irAEs among nivolumab users were maculopapular rash (13\%), erythema (4\%), hepatitis (3\%), and infusion-related reactions (3\%), while they were arthralgia (12\%), hypothyroidism (8\%), and hyperglycemia (6\%), among pembrolizumab users. 


\begin{abstract}
Conclusion: Especially the combination therapies tend to elevate the incidence of potential irAEs. Clinicians should be vigilant about irAEs following combination therapy as well as gastrointestinal and skin irAEs following ipilimumab therapy, in addition to being aware of potential irAEs leading to hyperglycemia, thyroid, hepatic, and musculoskeletal disorders following nivolumab and pembrolizumab therapy.
\end{abstract}

Keywords: immune checkpoint inhibitors, ipilimumab, nivolumab, pembrolizumab, incidence, immune-related adverse events, advanced, melanoma

\section{BACKGROUND}

Melanoma is a type of skin cancer, with increasing in incidence over the past several years. It is estimated to be the fifth most common cancer in the USA in 2019 and was the 21st most common cancer worldwide in $2018(1,2)$. Surgery is the standard primary treatment for melanoma. However, pharmacotherapy choices for patients with advanced melanoma have greatly expanded over the last few years, including the use of immune checkpoints inhibitors (ICIs) that target the cytotoxic T lymphocyte antigen-4 (CTLA-4) and programmed death-1 (PD-1) pathways (3). The US Food and Drug Administration (FDA) approved ipilimumab as the first ICI (anti-CTLA-4) therapy for advanced melanoma in 2011, followed by the antiPD-1 drugs nivolumab and pembrolizumab in 2014 (4-6). The combined use of anti-CTLA- 4 and anti-PD-1 drugs provides better clinical benefits over monotherapies, leading to the FDAapproved combination regimen of nivolumab and ipilimumab in 2015 (7).

The clinical benefits of ICIs are associated with a spectrum of adverse events (AEs) owing to the activation of the immune system that can affect healthy tissues of the body organs. These immune-related adverse events (irAEs) require close monitoring, use of corticosteroids and infliximab, holding the ICIs, or discontinuation of the drugs in case of severe irAEs such as diarrhea and colitis $(8-11)$. The reported incidence of irAEs is higher after anti-CTLA-4 treatment (90\%) than after anti-PD-1 treatment (70\%) across several types of advanced cancer (11), and the rates may vary based on the cancer type (12-16).

The incidences of irAEs owing to anti-CTLA-4 or antiPD-1 monotherapy as well as those owing to concomitant or sequential combination of these drugs are not wellestimated for advanced melanoma. Therefore, the aim of systematic review and meta-analysis was to profile the incidence of potential irAEs associated with mono- and combination therapies of ipilimumab, nivolumab, and pembrolizumab in advanced melanoma.

\section{METHODS}

\section{Search Strategy}

To identify eligible trials, we performed a comprehensive search of Medline, Embase, and Cochrane library from inception until October 30, 2018 (Table S1). We further searched clinicaltrials.gov as well as the websites of regulatory bodies in the USA (FDA), Europe [the European Medicines Agency (EMA)],
Australia [Therapeutic Goods Administration (TGA)], and Japan [Pharmaceuticals and Medical Devices Agency (PMDA)]. In addition, we screened the references of published reviews, metaanalyses, and relevant trials to include any related citation. We have reported this systemic review and meta-analysis following the Cochrane recommendations for the preferred reporting items for systematic reviews and meta-analyses (PRISMA) (17).

\section{Inclusion and Exclusion Criteria}

We included phase 1-3 clinical trials reporting any treatmentrelated AEs that could potentially be classified as irAEs following the use of anti-CTLA-4 or anti-PD-1 as monotherapy or in any concomitant or sequential combination of these agents in patients with advanced melanoma. We excluded trials reported in a non-English language, those restricted to uveal melanoma or pediatric populations, and those in the settings of compassionate care and expanded access programs or adjuvant therapy. We selected the most recently updated safety data for each included trial.

\section{Outcome Measures}

The primary outcome of interest was the incidence of potential irAEs across all regimens and doses studied in the trials. We obtained these events across all included trials if an $\mathrm{AE}$ was reported in any trial as "irAE", "AE of special interest," "selected treatment-related AE of special interest," or listed in the clinical practice guidelines for managing toxicities related to immunotherapies published by the European Society for Medical Oncology (ESMO), the American Society of Clinical Oncology (ASCO), and the National Comprehensive Cancer Network (NCCN) (8-10). We used the highest incidence of a potential irAE to estimate the overall incidence of irAEs if the information was not given in the included trials. The secondary outcome was the incidence of potential irAEs for the FDA-approved doses of anti-CTLA-4 and anti-PD-1 therapies.

\section{Selection of Studies and Data Extraction}

We used the citation management software Endnote ${ }^{\circledR}$ (version X7.3; Clarivate Analytics, Philadelphia, PA, USA) in the selection process. One reviewer (A.A.) removed the duplicates, after which, two reviewers (A.A. and A.M.) independently performed the selection in two stages: (1) screening of the title and abstract of each citation; and (2) reviewing the full text of the retained articles. During each stage, disagreements were 


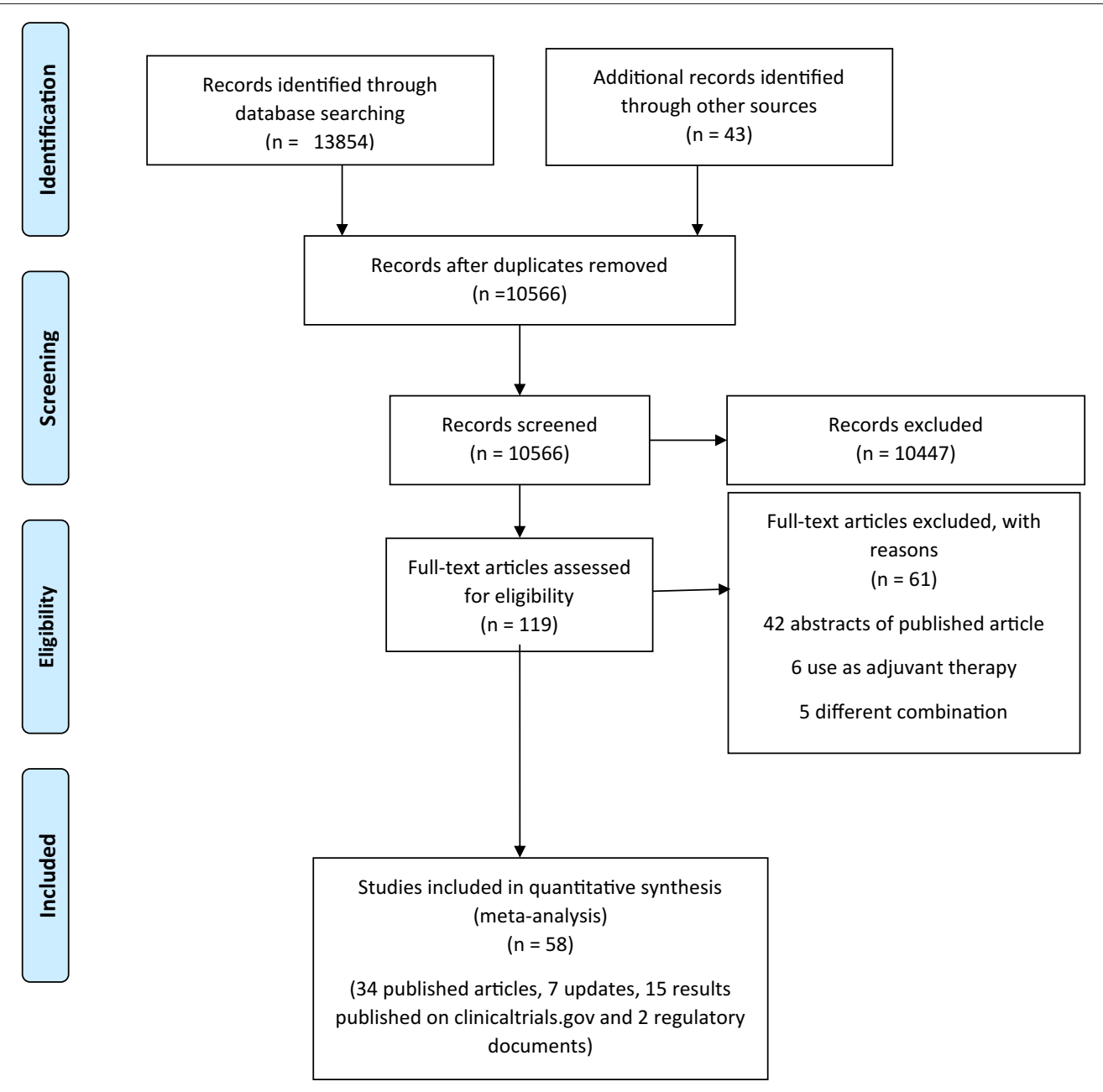

FIGURE 1 | Preferred reporting items for systematic reviews and meta-analyses (PRISMA) flow diagram.

resolved by consensus or escalated to a third reviewer (I.A.). One reviewer (A.A.) extracted the data into a Microsoft Excel file, including the trial title, trial phase, the total number of patients, and the number of patients who experienced AEs (all grades and grade $\geq 3$ ). A second reviewer (A.M.) verified the extraction file.

\section{Assessment of the Quality of the Included} Trials

To assess the risk of bias in the included trials, we used the Cochrane Collaboration risk of bias assessment tool for randomized trials and the Newcastle-Ottawa Scale for nonrandomized trials $(18,19)$. Two investigators (A.A. and A.M.) performed the quality assessment of the trials and resolved any disagreement by internal discussion or escalation to a third investigator (I.A.).

\section{Statistical Analysis}

We performed random effect meta-analyses of binomial data to estimate the incidence of potential irAEs. We employed the Freeman-Tukey double arcsine transformation to stabilize the variance and avoid overestimation bias that might be introduced by continuity correction for zero events (20). The 95\% confidence interval $(95 \% \mathrm{CI})$ was estimated by using the score test owing to its better performance than the Wald and Exact CIs (21). We assessed heterogeneity based on the $I^{2}$ statistic and conducted subgroup analyses when heterogeneity of $I^{2}>75 \%$ was observed. We evaluated publication bias using the funnel plot and Egger's test. Meta-analyses were performed using $\mathrm{R}$ software version 3.4.3 ("meta" package).

\section{RESULTS}

\section{Search Results}

We initially identified 13,897 records through database searching and additional sources. After removing the duplicates, screening the titles and abstracts, and applying the inclusion and exclusion criteria, we retained 58 records $(34$ published articles, 7 updates, 15 results published on clinicaltrials.gov, 
and 2 regulatory documents) of 35 unique trials (Figure 1) (22-62). $1,2,3,4,5,6,7,8,9,10,11,12,13,14,15,16,17$

\section{Characteristics of the Included Trials}

The 35 trials included 6,331 advanced melanoma patients treated with anti-CTLA-4 or anti-PD-1 monotherapy or combination therapy: 2,726 received ipilimumab in 21 studies $(23,29,39-$ $53,56)^{12},(61,62), 1,019$ received nivolumab in 7 studies $(23$, $24,34,36-38,60), 1,632$ received pembrolizumab in 5 studies $(28-30,33,57), 630$ received the concomitant combination of nivolumab and ipilimumab in 5 studies $(23-25,54,56), 103$ patients received the sequential combination of ipilimumab followed by nivolumab in 2 studies including 33 patients previously treated with ipilimumab then used nivolumab therapy

\footnotetext{
${ }^{1}$ Study of Nivolumab Given Sequentially With Ipilimumab in Subjects With Advanced or Metastatic Melanoma (CheckMate 064). Available online at: https:// ClinicalTrials.gov/show/NCT01783938

${ }^{2}$ Study of Nivolumab (BMS-936558) Plus Ipilimumab Compared With Ipilimumab Alone in the Treatment of Previously Untreated, Unresectable, or Metastatic Melanoma. Available online at: https://ClinicalTrials.gov/show/ NCT01927419.

${ }^{3}$ Study of Pembrolizumab (MK-3475) in Participants With Advanced Melanoma (MK-3475-041/KEYNOTE-041). Available online at: https:/ClinicalTrials.gov/ show/NCT02180061

${ }^{4}$ Study to Evaluate the Safety and Efficacy of Two Different Dosing Schedules of Pembrolizumab (MK-3475) Compared to Ipilimumab in Participants With Advanced Melanoma (MK-3475-006/KEYNOTE-006). Available online at: https://ClinicalTrials.gov/show/NCT01866319

${ }^{5}$ Study of Pembrolizumab (MK-3475) Versus Chemotherapy in Participants With Advanced Melanoma (P08719/KEYNOTE-002). Available online at: https:// ClinicalTrials.gov/show/NCT01704287

${ }^{6}$ A Study to Compare BMS-936558 to the Physician's Choice of Either Dacarbazine or Carboplatin and Paclitaxel in Advanced Melanoma Patients That Have Progressed Following Anti-CTLA-4 Therapy (CheckMate 037). Available online at: https://ClinicalTrials.gov/show/NCT01721746

${ }^{7}$ Study of Nivolumab (BMS-936558) Compared With Dacarbazine in Untreated, Unresectable, or Metastatic Melanoma. Available online at: https://ClinicalTrials. gov/show/NCT01721772

${ }^{8}$ Evaluation of Tumor Response to Ipilimumab in the Treatment of Melanoma
} With Brain Metastases. Available online at: https://ClinicalTrials.gov/show/ NCT00623766

${ }^{9}$ Ipilimumab With or Without Talimogene Laherparepvec in Unresected Melanoma. Available online at: https://ClinicalTrials.gov/show/NCT01740297

${ }^{10}$ Phase 3 Trial in Subjects With Metastatic Melanoma Comparing $3 \mathrm{mg} / \mathrm{kg}$ Ipilimumab Versus $10 \mathrm{mg} / \mathrm{kg}$ Ipilimumab. Available online at: https:// ClinicalTrials.gov/show/NCT01515189

${ }^{11}$ Drug-Drug Interaction - 3 Arm - Carboplatin/Paclitaxel, Dacarbazine. Available online at: https://ClinicalTrials.gov/show/NCT00796991

${ }^{12}$ Comparison of Ipilimumab Manufactured by 2 Different Processes in Participants With Advanced Melanoma. Available online at: https://ClinicalTrials. gov/show/NCT00920907

${ }^{13}$ MDX-010 Antibody, MDX-1379 Melanoma Vaccine, or MDX-010/MDX-1379 Combination Treatment for Patients With Unresectable or Metastatic Melanoma. Available online at: https://ClinicalTrials.gov/show/NCT00094653

${ }^{14}$ Phase 2 Study of Ipilimumab in Japanese Advanced Melanoma Patients. Available online at: https://ClinicalTrials.gov/show/NCT01990859

${ }^{15}$ Ipilimumab With or Without Sargramostim in Treating Patients With Stage III or Stage IV Melanoma That Cannot Be Removed by Surgery. Available online at: https://ClinicalTrials.gov/show/NCT01134614

${ }^{16}$ Goods Therapeutic Administration. Australian Public Assessment Report for Ipilimumab 2011. Available online at: https:/www.tga.gov.au/sites/default/files/ auspar-yervoy.pdf.

${ }^{17}$ Pharmaceutical and Mdical Devices Agency. Report on Deliberation Results for Ipilimumab 2015. Available online at: https://www.pmda.go.jp/files/000215223.pdf every 2 weeks for up to 48 doses within $4-12$ weeks from the ipilimumab treatment, and 70 patients treated with ipilimumab every 3 weeks for 4 doses followed 3 weeks later by nivolumab therapy every 2 weeks up to progression, unacceptable toxicity or withdrawal from the study $(22,54), 68$ patients received the sequential combination of nivolumab followed by ipilimumab in 1 study in which patients treated with nivolumab every 2 weeks for 6 doses then followed 2 weeks later by ipilimumab therapy every 3 weeks for 4 doses, and subsequently followed by nivolumab therapy every 2 weeks up to progression, unacceptable toxicity or withdrawal from study (22), and 153 received the combination of pembrolizumab and ipilimumab in 1 study (26). Approximately 50\% of the patients included in these trials had received prior systemic therapy before starting the assigned ICI therapy. Of the trials included, 11 were phase 1 , 18 phase 2, 2 phase $1 / 2$, and 6 phase 3 studies (Table 1 ).

\section{Quality of the Included Trials and Publication Bias}

Our quality assessment of the 19 randomized trials revealed that 10 trials were not blinded (Figure 2). All the non-randomized trials (16 trials) were open-label, and most of them were single arm (Table S2). Open-label trials are susceptible to detection bias and may lead to over-reporting of irAEs. Results from funnel plots and the Egger's test did not show evidence of publication bias (Figures S1-S16).

\section{Incidence of Potential irAEs}

The incidence of all grade and grade $\geq 3$ potential irAEs owing to all the doses or FDA-approved doses of ICIs based on system disorders is summarized in Tables $\mathbf{2}-\mathbf{5}$.

\section{Blood and Lymphatic System Disorders}

All-grade lymphopenia was more likely to occur after pembrolizumab, with an incidence of $4.8 \%$; however, the incidence of grade $\geq 3$ lymphopenia was $0.8 \%$ in nivolumab users. In the FDA-approved doses, the incidence of all-grade and grade $\geq 3$ lymphopenia reached 6.8 and $2.2 \%$ respectively, in nivolumab users.

\section{Cardiac Disorders}

The combination of ipilimumab and nivolumab resulted in an incidence of $1.1 \%$ of all-grade ventricular arrhythmia. Moreover, the incidence of ventricular arrhythmia and pericarditis (allgrade and grade $\geq 3$ ) was $0.4 \%$ in nivolumab users.

\section{Endocrine Disorders}

The incidence of all-grade and grade $\geq 3$ potential endocrine irAEs frequently occurred after the combination of pembrolizumab and ipilimumab or nivolumab and ipilimumab. Hyperthyroidism, hypothyroidism, hypophysitis, and thyroiditis were the most frequent potential endocrine irAEs. After antiPD-1 therapy (nivolumab or pembrolizumab), the incidence of all-grade hyperthyroidism and hypothyroidism was 3.0-3.4 and $7.0-8.3 \%$, respectively. After ipilimumab treatment, the observed incidence of all-grade and grade $\geq 3$ hypophysitis and all-grade and grade $\geq 3$ hypopituitarism was $4.1,2.1,1.5$, 
TABLE 1 | Characteristics of included studies.

Trial Phase Treatment arm

Phase Treatment arm

Pembrolizumab 2 mg/kgQ3wks (up to 2 years) + ipilimumab $1 \mathrm{mg} / \mathrm{kgQ} 3 w \mathrm{ks}$ for 4 doses

KEYNOTE-029

Pembrolizumab

KEYNOTE-001

1

Pembrolizumab 2 mg/kgQ3wks

Pembrolizumab 10 mg/kgQ3wks

Pembrolizumab 10 mg/kgQ2wks

Pembrolizumab 2 mg/kgQ3wks

Pembrolizumab 10 mg/kgQ3wks

Pembrolizumab 10 mg/kgQ2wks

KEYNOTE-041

1 Pembrolizumab 2 mg/kgQ3wks

NCT02085070

2 Pembrolizumab $10 \mathrm{mg} / \mathrm{kgQ} 2 \mathrm{wks}$

KEYNOTE-002

2 Pembrolizumab 2 mg/kgQ3wks

Pembrolizumab 10 mg/kgQ3wks

KEYNOTE-006

3 Pembrolizumab 10 mg/kgQ2wks

Pembrolizumab 10 mg/kgQ3wks

\section{Nivolumab and Ipilimumab (concomitant)}

CA209-004

Nivolumab $0.3 \mathrm{mg} / \mathrm{kg}+$ ipilimumab $3 \mathrm{mg} / \mathrm{kgQ} 3 w \mathrm{ks}$ for 4 doses then nivolumab $0.3 \mathrm{mg} / \mathrm{kgQ} 3 w \mathrm{ks}$ for 4 doses then nivolumab $0.3 \mathrm{mg} / \mathrm{kg}+$ ipilimumab 3 $\mathrm{mg} / \mathrm{kgQ} 12 \mathrm{wks}$ for 8 doses

Nivolumab $1 \mathrm{mg} / \mathrm{kg}+$ ipilimumab $3 \mathrm{mg} / \mathrm{kgQ} 3 w \mathrm{ks}$ for 4 doses then nivolumab $1 \mathrm{mg} / \mathrm{kgQ} 3 w \mathrm{ks}$ for 4 doses then nivolumab $1 \mathrm{mg} / \mathrm{kg}+$ ipilimumab 3 $\mathrm{mg} / \mathrm{kgQ12}$ wks for 8 doses

Nivolumab 3 mg/kg + ipilimumab 1 mg/kgQ3wks for 4 doses then nivolumab $3 \mathrm{mg} / \mathrm{kgQ} 3 \mathrm{wks}$ for 4 doses then nivolumab $3 \mathrm{mg} / \mathrm{kg}+$ ipilimumab 1 $\mathrm{mg} / \mathrm{kgQ12wks}$ for 8 doses

Nivolumab 3 mg/kg+ ipilimumab 3 mg/kgQ3wks for 4 doses then nivolumab $3 \mathrm{mg} / \mathrm{kgQ} 3 \mathrm{wks}$ for 4 doses then nivolumab $3 \mathrm{mg} / \mathrm{kg}+$ ipilimumab 3

$\mathrm{mg} / \mathrm{kgQ12}$ wks for 8 doses

Nivolumab $1 \mathrm{mg} / \mathrm{kg}+$ ipilimumab $3 \mathrm{mg} / \mathrm{kgQ} 3 w \mathrm{ks}$ for 4 doses then nivolumab $1 \mathrm{mg} / \mathrm{kgQ} 3$ wks for up to 48 doses

CheckMate-069 2 Nivolumab $1 \mathrm{mg} / \mathrm{kg}+$ ipilimumab $3 \mathrm{mg} / \mathrm{kgQ3}$ wks for 4 doses then nivolumab $3 \mathrm{mg} / \mathrm{kgQ} 2 \mathrm{wks}$

CheckMate-204 2 Nivolumab $1 \mathrm{mg} / \mathrm{kg}+$ ipilimumab $3 \mathrm{mg} / \mathrm{kgQ3wks}$ for 4 doses then nivolumab $3 \mathrm{mg} / \mathrm{kgQ} 2 \mathrm{wks}$

NCT02374242

2 Nivolumab $1 \mathrm{mg} / \mathrm{kg}+$ ipilimumab $3 \mathrm{mg} / \mathrm{kgQ} 3 w \mathrm{ks}$ for 4 doses then nivolumab $3 \mathrm{mg} / \mathrm{kgQ} 2 \mathrm{wks}$

CheckMate-067 $3 \quad$ Nivolumab $1 \mathrm{mg} / \mathrm{kg}+$ ipilimumab $3 \mathrm{mg} / \mathrm{kgQ} 3 \mathrm{wks}$ for 4 doses then nivolumab $3 \mathrm{mg} / \mathrm{kgQ} 2 \mathrm{wks}$

Nivolumab and Ipilimumab (Sequential of ipilimumab then nivolumab)

CA209-004

1 Ipilimumab followed by nivolumab $1 \mathrm{mg} / \mathrm{kg}$ (within 4-12wks of last dose of ipilimumab
Control arm

Treatment Previous treatment (N)

(N)
NA

153

NA

NA

NA

chemotherapy

ipilimumab 3 mg/kgQ3wks for 4 doses

NA

Ipilimumab 3 mg/kgQ3wks for 4 doses

NA

Nivolumab 3 mg/kgQ2wks

Nivolumab 3 mg/kgQ2wks

OR ipilimumab 3

$\mathrm{mg} / \mathrm{kgQ} 3$ wks for 4 doses
20 prior systemic therapy

342 prior ipilimumab therapy 342 prior systemic therapy

172

81

141

99

42

23

178

179

278

277

prior ipilimumab therapy 42 prior systemic therapy systemic therapy systemic therapy

178 prior ipilimumab therapy 178 prior systemic therapy

179 prior ipilimumab therapy 178 prior systemic therapy

0 prior ipilimumab therapy 96 prior systemic therapy systemic therapy
13 prior ipilimumab therapy 16 prior

0 prior ipilimumab therapy 91 prior
0 prior ipilimumab therapy 30 prior
0 prior systemic therapy

0 prior systemic therapy

16 prior systemic therapy

8 prior systemic therapy

17
33 prior ipilimumab therapy 33 prior systemic therapy 
TABLE 1 | Continued

\begin{tabular}{|c|c|c|c|c|c|}
\hline Trial & Phase & Treatment arm & Control arm & $\begin{array}{l}\text { Treatment } \\
\text { (N) }\end{array}$ & Previous treatment $(\mathrm{N})$ \\
\hline & & $\begin{array}{l}\text { Ipilimumab followed by nivolumab } 3 \mathrm{mg} / \mathrm{kg} \text { (within } \\
4-12 \text { wks of last dose of ipilimumab }\end{array}$ & & 16 & \\
\hline CA209-064 & 2 & $\begin{array}{l}\text { Induction period } 1 \text { (ipilimumab } 3 \text { mg/kgQ3wks up to } \\
4 \text { doses during weeks 1-13). Induction period } 2 \\
\text { (nivolumab } 3 \mathrm{mg} / \mathrm{kg} \text { Q2wks up to } 6 \text { doses during } \\
\text { weeks } 13-25 \text { ) Continuation period (nivolumab } 3 \\
\text { mg/kgQ2wks from week } 25 \text { till completion of } 2 \\
\text { years) }\end{array}$ & $\begin{array}{l}\text { Nivolumab followed by } \\
\text { ipilimumab }\end{array}$ & 70 & 0 prior systemic therapy \\
\hline \multicolumn{6}{|c|}{ Nivolumab and Ipilimumab (Sequential of nivolumab then ipilimumab) } \\
\hline CA209-064 & 2 & $\begin{array}{l}\text { Induction period } 1 \text { (nivolumab } 3 \mathrm{mg} / \mathrm{kgQ} 2 \text { wks up to } \\
6 \text { doses during weeks } 1-13 \text { ). Induction period } 2 \\
\text { (ipilimumab } 3 \mathrm{mg} / \mathrm{kg} \text { Q3wks up to } 4 \text { doses during } \\
\text { weeks } 13-25 \text { ) Continuation period (nivolumab } 3 \\
\text { mg/kgQ2wks from week } 25 \text { till completion of } 2 \\
\text { years) }\end{array}$ & $\begin{array}{l}\text { Ipilimumab followed by } \\
\text { nivolumab }\end{array}$ & 68 & 0 prior systemic therapy \\
\hline CA209-006 & $1 / 2$ & $\begin{array}{l}\text { Nivolumab } 3 \mathrm{mg} / \mathrm{kgQ} 2 \text { wks for } 24 \text { weeks then } \\
\text { Q12wks for up to } 2 \text { years }\end{array}$ & NA & 61 & $\begin{array}{l}61 \text { prior ipilimumab therapy } \\
31 \text { prior systemic therapy }\end{array}$ \\
\hline JapicCTI-142533 & 2 & Nivolumab 3 mg/kgQ2wks & NA & 23 & 0 prior systemic therapy \\
\hline NCT02374242 & 2 & Nivolumab 3 mg/kgQ2wks & $\begin{array}{l}\text { Nivolumab } 1 \mathrm{mg} / \mathrm{kg}+ \\
\text { Ipilimumab } 3 \mathrm{mg} / \mathrm{kgQ} 3 w \mathrm{ks} \\
\text { for } 4 \text { doses then Nivolumab } \\
3 \mathrm{mg} / \mathrm{kgQ} 2 \text { wks }\end{array}$ & 41 & 18 prior systemic therapy \\
\hline CheckMate 066 & 3 & Nivolumab 3 mg/kgQ2wks & Dacarbazine & 206 & 33 prior systemic therapy \\
\hline CheckMate 067 & 3 & Nivolumab 3 mg/kgQ2wks & $\begin{array}{l}\text { Nivolumab +ipilimumab OR } \\
\text { ipilimumab } 3 \mathrm{mg} / \mathrm{kgQ} 3 \mathrm{wks} \\
\text { for } 4 \text { doses }\end{array}$ & 313 & 0 prior systemic therapy \\
\hline CA184-078 & 1 & Ipilimumab 10 mg/kgQ3wks for 4 doses + placebo & $\begin{array}{l}\text { Ipilimumab + dacarbazine } \\
\text { OR ipilimumab + } \\
\text { paclitaxel+carboplatin }\end{array}$ & 20 & 0 prior systemic therapy \\
\hline CA184-087 & 1 & Ipilimumab 10 mg/kgQ3wks for 4 doses & NA & 75 & \\
\hline CA184-013 & 2 & Ipilimumab 3 mg/kgQ3wks for 4 doses & Ipilimumab + dacarbazine & 39 & $\begin{array}{l}0 \text { prior chemotherapy } 19 \text { prior } \\
\text { immunotherapy }\end{array}$ \\
\hline \multirow[t]{2}{*}{ CA184-169 } & 3 & Ipilimumab 3 mg/kgQ3wks for 4 doses & NA & 362 & 205 prior systemic therapy \\
\hline & & Ipilimumab 10 mg/kgQ3wks for 4 doses & & 364 & 206 prior systemic therapy \\
\hline CA184-396 & 2 & Ipilimumab 3 mg/kgQ3wks for 4 doses & NA & 20 & 16 prior systemic therapy \\
\hline CheckMate 067 & 3 & Ipilimumab 3 mg/kgQ3wks for 4 doses & $\begin{array}{l}\text { Nivolumab +ipilimumab OR } \\
\text { nivolumab } 3 \text { mg/kgQ2wks }\end{array}$ & 311 & 0 prior systemic therapy \\
\hline CheckMate-069 & 2 & Ipilimumab 3 mg/kgQ3wks for 4 doses & $\begin{array}{l}\text { Nivolumab } 1 \mathrm{mg} / \mathrm{kg}+ \\
\text { ipilimumab } 3 \mathrm{mg} / \mathrm{kgQ} 3 w \mathrm{ks} \\
\text { for } 4 \text { doses then nivolumab } \\
3 \mathrm{mg} / \mathrm{kgQ} 2 \mathrm{wks}\end{array}$ & 46 & 0 prior systemic therapy \\
\hline \multirow[t]{2}{*}{ KEYNOTE-006 } & 3 & Ipilimumab 3 mg/kgQ3wks for 4 doses & $\begin{array}{l}\text { Pembrolizumab } 10 \\
\mathrm{mg} / \mathrm{kgQ} 2 \mathrm{wks}\end{array}$ & 256 & $\begin{array}{l}0 \text { prior ipilimumab therapy } 97 \text { prior } \\
\text { systemic therapy }\end{array}$ \\
\hline & & & $\begin{array}{l}\text { pembrolizumab } 10 \\
\text { mg/kgQ3wks }\end{array}$ & & \\
\hline
\end{tabular}


TABLE 1 | Continued

\begin{tabular}{|c|c|c|c|c|c|}
\hline Trial & Phase & Treatment arm & Control arm & $\begin{array}{l}\text { Treatm } \\
\text { (N) }\end{array}$ & Previous treatment (N) \\
\hline \multirow[t]{3}{*}{$\begin{array}{l}\text { CA184-001 } \\
(\text { MDX010-15) }\end{array}$} & $1 / 2$ & $\begin{array}{l}\text { Ipilimumab } 3 \mathrm{mg} / \mathrm{kg} \text { or } 2.8 \text { or } 5 \text { mg/kg on Days } 1 \text {, } \\
57 \text {, and } 85\end{array}$ & NA & 34 & 31 prior systemic therapy \\
\hline & & Single dose of ipilimumab $7.5,10,15$, or 20 mg/kg & & 30 & 21 prior systemic therapy \\
\hline & & Ipilimumab 10 mg/kgQ3wks for 4 doses & & 24 & 18 prior systemic therapy \\
\hline NCT01134614 & 2 & $\begin{array}{l}\text { Ipilimumab } 10 \text { mg/kgQ3wks for } 4 \text { doses then } \\
\text { Q12wks }\end{array}$ & Ipilimumab + sargramostim & 120 & 54 prior systemic therapy \\
\hline NCT01216696 & 2 & $\begin{array}{l}\text { Ipilimumab } 10 \mathrm{mg} / \mathrm{kgQ} 3 w \mathrm{ks} \text { for } 4 \text { doses then } \\
\text { Q12wks }\end{array}$ & NA & 25 & 19 prior systemic therapy \\
\hline NCT01355120 & 2 & Ipilimumab 3 mg/kgQ3wks for 4 doses & NA & 103 & 103 prior systemic therapy \\
\hline NCT01740297 & 2 & Ipilimumab 3 mg/kgQ3wks for 4 doses & $\begin{array}{l}\text { Ipilimumab+ talimogene } \\
\text { laherparepvec }\end{array}$ & 95 & 29 prior anticancer therapy \\
\hline \multirow[t]{2}{*}{ CA184-004 } & 2 & Ipilimumab 3 mg/kgQ3wks for 4 doses then Q12 & NA & 40 & 29 prior systemic therapy \\
\hline & & $\begin{array}{l}\text { Ipilimumab } 10 \mathrm{mg} / \mathrm{kgQ} 3 w \mathrm{ks} \text { for } 4 \text { doses then } \\
\text { Q12wks }\end{array}$ & & 42 & 33 prior systemic therapy \\
\hline CA184-007 & 2 & Ipilimumab 10 mg/kgQ3wks for 4 doses + placebo & Ipilimumab + budesonide & 57 & 41 prior systemic therapy \\
\hline CA184-008 & 2 & $\begin{array}{l}\text { Ipilimumab } 10 \mathrm{mg} / \mathrm{kgQ} 3 w \mathrm{ks} \text { for } 4 \text { doses then } \\
\text { Q12wks }\end{array}$ & NA & 155 & 155 prior systemic therapy \\
\hline \multirow[t]{3}{*}{ CA184-022 } & 2 & $\begin{array}{l}\text { Ipilimumab } 0.3 \text { mg/kgQ3wks for } 4 \text { doses then } \\
\text { Q12wks }\end{array}$ & NA & 72 & 72 prior systemic therapy \\
\hline & & $\begin{array}{l}\text { Ipilimumab } 3 \mathrm{mg} / \mathrm{kgQ} 3 w \mathrm{ks} \text { for } 4 \text { doses then } \\
\text { Q12wks }\end{array}$ & & 71 & 71 prior systemic therapy \\
\hline & & $\begin{array}{l}\text { Ipilimumab } 10 \mathrm{mg} / \mathrm{kgQ} 3 w \mathrm{ks} \text { for } 4 \text { doses then } \\
\text { Q12wks }\end{array}$ & & 71 & 71 prior systemic therapy \\
\hline MDX010-20 & 3 & $\begin{array}{l}\text { Ipilimumab } 10 \text { mg/kgQ3wks for } 4 \text { doses then } \\
\text { Q12wks if patients met the re-induction criteria }\end{array}$ & $\begin{array}{l}\text { Ipilimumab + gp100 or } \\
\text { gp100 alone }\end{array}$ & 131 & 131 prior systemic therapy \\
\hline CA184-042 & 2 & $\begin{array}{l}\text { Ipilimumab } 10 \text { mg/kgQ3wks for } 4 \text { doses then } \\
\text { Q12wks }\end{array}$ & Ipilimumab+ corticosteroids & 51 & 40 prior systemic therapy \\
\hline
\end{tabular}

$N$, number of patients.

and $1.0 \%$, respectively. The estimated incidence of all-grade adrenal insufficiency and thyroiditis was $1.6 \%$ after nivolumab. On administering the FDA-approved dose of ipilimumab, the incidence of all-grade hypophysitis was $2.6 \%$ and that of grade $\geq 3$ hypophysitis was $1.2 \%$, with only $<1 \%$ of patients developed hypopituitarism.

\section{Eye Disorders}

The incidence of all-grade uveitis was $2.6 \%$ after the combination of ipilimumab and pembrolizumab and $2.0 \%$ after the combination therapy with ipilimumab and nivolumab, but the incidence after monotherapies was $<1 \%$.

\section{Gastrointestinal Disorders}

The ICI combinations (ipilimumab and nivolumab or ipilimumab and pembrolizumab) resulted in the most frequent incidence of colitis (all-grade and grade $\geq 3$ ), pancreatitis (all-grade and grade $\geq 3$ ), diarrhea (all-grade), and autoimmune colitis (grade $\geq 3$ ). Ipilimumab use was associated with developing diarrhea (all-grade in $29.2 \%$ and grade $\geq 3$ in $5.9 \%$ ) and colitis (all-grade in $8.0 \%$ and grade $\geq 3$ in $5.4 \%$ ).

\section{General Disorders}

All-grade infusion-related reaction was observed in $7.4 \%$ of users of the sequential therapy of nivolumab followed by ipilimumab and in $3.9 \%$ of users of the concomitant therapy of ipilimumab and pembrolizumab. However, among the monotherapies, nivolumab resulted in an incidence of $3.0 \%$ of all-grade infusionrelated reactions.

\section{Hepatobiliary Disorders}

Hepatitis was the most common frequent potential irAE reported after ICI monotherapy or combination therapies. The incidence of all-grade and grade $\geq 3$ hepatitis after the combination of pembrolizumab and ipilimumab was 9.8 and 5.9\%, respectively; the corresponding values after the combination of nivolumab and ipilimumab were 4.9 and $3.5 \%$, and those after the sequential therapy of nivolumab followed by ipilimumab were 4.4 and $4.4 \%$. Nivolumab monotherapy resulted in an incidence of $3.0 \%$ of all-grade hepatitis.

\section{Immune System Disorders}

The incidence of all-grade hypersensitivity after nivolumab alone or in combination with ipilimumab including the FDA-approved doses was 2.0 and $2.9 \%$, respectively.

\section{Infections}

The incidence of all-grade and grade $\geq 3$ aseptic meningitis and encephalitis was $1.5 \%$ after sequential therapy of nivolumab 

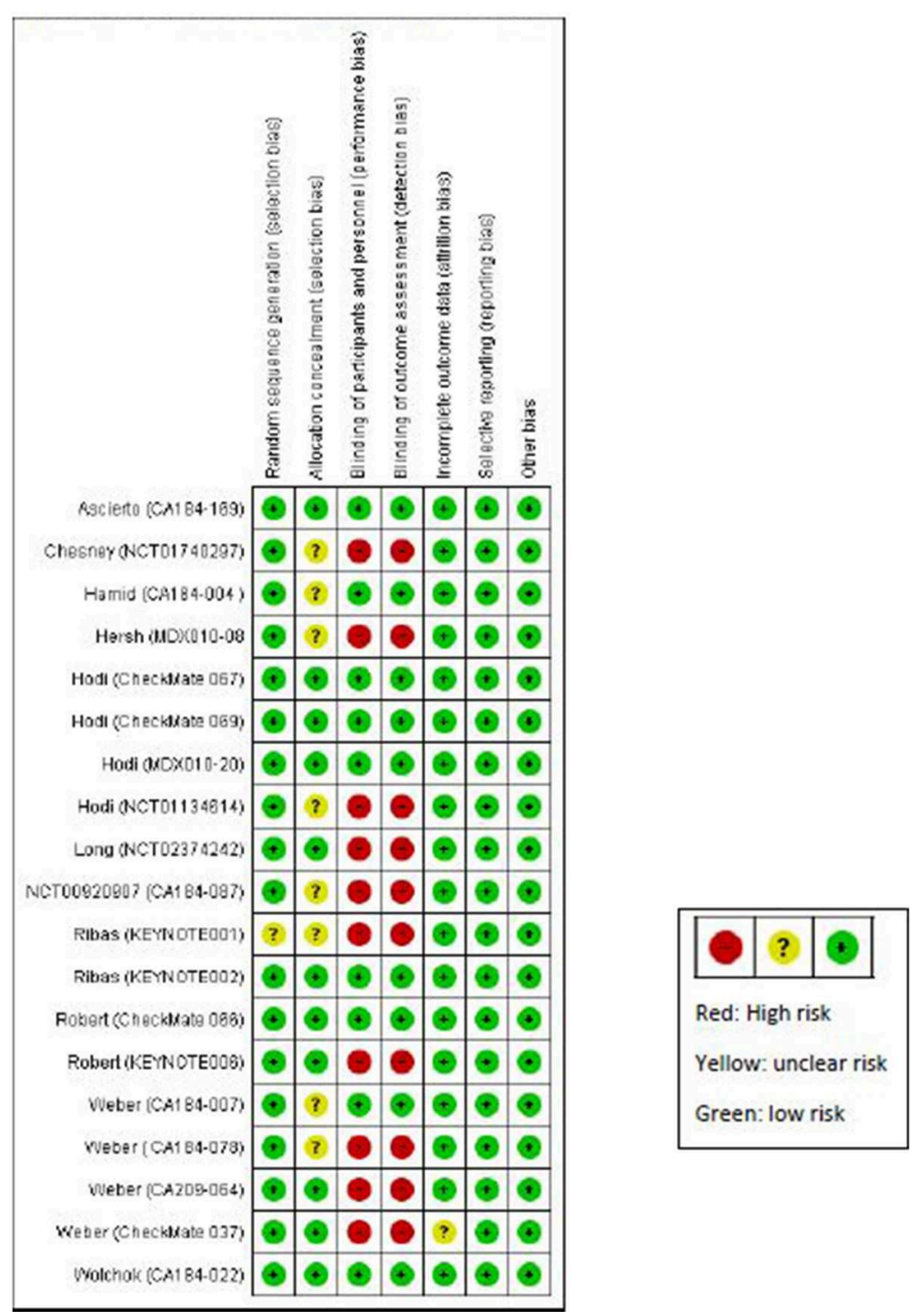

FIGURE 2 | Quality assessment of randomized studies using Cochrane Collaboration risk of bias assessment tool for randomized trials.

followed by ipilimumab. Ipilimumab users had an incidence of $1.7 \%$ for all-grade conjunctivitis.

\section{Investigations}

The incidences of elevated or abnormal levels of most enzymes and hormones (all-grade) were reported at higher frequency after the combination of ICIs. The elevated enzymes and hormones that were reported frequently following the combination therapies included alanine aminotransferase (ALT), aspartate aminotransferase (AST), amylase, lipase, blood alkaline phosphatase, and blood bilirubin. The incidence of all-grade abnormal thyroid stimulating hormone (TSH) was 3.3 and $6.7 \%$ after concomitant combinations of ipilimumab and pembrolizumab or ipilimumab and nivolumab, respectively; the incidence of all-grade and grade $\geq 3$ elevated gammaglutamyl transferase (GGT) was, respectively, 7.2 and $1.3 \%$ after concomitant ipilimumab and pembrolizumab and 3.3 and $1.0 \%$ after concomitant ipilimumab and nivolumab. Among the monotherapies, after ipilimumab, the incidence of all-grade elevated ALT was 3.9\%; after nivolumab, the incidence of all-grade and grade $\geq 3$ elevated amylase was 5.8 and $1.5 \%$, respectively, with an incidence of all-grade elevated blood alkaline phosphatase of $3.8 \%$ and an incidence of abnormal TSH of $2.8 \%$. After pembrolizumab, the incidence of all-grade AST was $4.9 \%$, that of all-grade elevated blood bilirubin was $1.6 \%$, and that of all-grade elevated transaminases was $1.2 \%$. For the FDAapproved doses, the combination of nivolumab and ipilimumab had the most frequent incidence of most all-grade and grade $\geq 3$ elevated or abnormal enzymes or hormones. The most frequent enzymes and hormones abnormalities associated with nivolumab use were all-grade and grade $\geq 3$ elevated lipase (3.1 and $2.2 \%$, respectively), all-grade and grade $\geq 3$ elevated amylase (5.8 and 
TABLE 2 | Incidence of All-grades Potential Immune-Related Adverse Events (irAEs) in Advanced Melanoma presented as $\%$ with $95 \%$ confidence interval, and $l^{2}$ for the $\%$ of heterogeneity ( $I^{2}$ not reported if the outcome was obtained from one study).

\begin{tabular}{|c|c|c|c|c|c|c|c|}
\hline irAEs & Ipilimumab & Nivolumab & Pembrolizumab & $\begin{array}{l}\text { Pembrolizumab } \\
\text { +Ipilimumab }\end{array}$ & $\begin{array}{c}\text { Nivolumab } \\
+ \text { Ipilimumab }\end{array}$ & $\begin{array}{c}\text { Ipilimumab } \\
\text { then } \\
\text { Nivolumab } \\
\text { (Sequential) }\end{array}$ & $\begin{array}{c}\text { Nivolumab } \\
\text { then } \\
\text { Ipilimumab } \\
\text { (Sequential) }\end{array}$ \\
\hline Number of studies & 21 & 7 & 5 & 1 & 5 & 2 & 1 \\
\hline Overall incidence & $\begin{array}{l}62.22 \text { [54.59; } \\
69.58], 93.0 \%\end{array}$ & $\begin{array}{l}49.39 \text { [36.17; } \\
62.67], 94.0 \%\end{array}$ & $\begin{array}{c}2300 \text { [17.00; } \\
29.00], 81.0 \%\end{array}$ & $\begin{array}{c}60.13[52.22 ; \\
\quad 67.55]\end{array}$ & $\begin{array}{l}83.00 \text { [65.00; } \\
95.00], 96.0 \%\end{array}$ & $\begin{array}{l}35.00 \text { [17.00; } \\
55.00], 73.9 \%\end{array}$ & $\begin{array}{c}47.00[36.00 \\
59.00]\end{array}$ \\
\hline \multicolumn{8}{|c|}{ Blood and lymphatic system disorders } \\
\hline Lymphopenia & $\begin{array}{c}0.98[0.00 ; 3.00] \\
14.3 \%\end{array}$ & $\begin{array}{c}4.01[0.87 ; 9.02] \\
66.6 \%\end{array}$ & $4.76[1.32 ; 15.79]$ & $N R$ & $N R$ & NR & NR \\
\hline \multicolumn{8}{|l|}{ Cardiac disorders } \\
\hline Pericarditis & 0.14 [0.02; 0.78] & 0.37 [0.07; 2.08] & 0.15 [0.03; 0.86] & NR & NR & NR & NR \\
\hline $\begin{array}{l}\text { Ventricular } \\
\text { arrhythmia }\end{array}$ & $0.00[0.00 ; 7.71]$ & $0.37[0.07 ; 2.08]$ & NR & NR & $1.06[0.19 ; 5.78]$ & NR & NR \\
\hline \multicolumn{8}{|l|}{ Endocrine disorders } \\
\hline $\begin{array}{l}\text { Acute } \\
\text { adrenocortical } \\
\text { insufficiency }\end{array}$ & $\begin{array}{c}0.14[0.00 ; 0.53] \\
0.0 \%\end{array}$ & $0.00[0.00 ; 1.21]$ & $0.28[0.05 ; 1.57]$ & $0.65[0.12 ; 3.61]$ & $0.32[0.06 ; 1.79]$ & NR & NR \\
\hline $\begin{array}{l}\text { Adrenal } \\
\text { insufficiency }\end{array}$ & $\begin{array}{c}0.67[0.28 ; 1.17] \\
0.00 \%\end{array}$ & $\begin{array}{c}1.68[0.16 ; 4.34] \\
68.1 \%\end{array}$ & $\begin{array}{c}0.33[0.03 ; 0.82] \\
0.0 \%\end{array}$ & $3.27[1.40 ; 7.42]$ & $\begin{array}{c}4.21[2.67 ; 6.04] \\
0.0 \%\end{array}$ & $\begin{array}{c}3.76[0.61 ; 8.71] \\
0.0 \%\end{array}$ & $8.82[4.11 ; 17.94]$ \\
\hline $\begin{array}{l}\text { Adrenocorticotropic } \\
\text { hormone } \\
\text { deficiency }\end{array}$ & $0.41[0.14 ; 1.21]$ & NR & NR & $0.65[0.12 ; 3.61]$ & NR & NR & NR \\
\hline $\begin{array}{l}\text { Autoimmune } \\
\text { thyroiditis }\end{array}$ & $\begin{array}{c}0.00[0.00 ; 0.16] \\
0.0 \%\end{array}$ & $0.32[0.06 ; 1.79]$ & NR & NR & $\begin{array}{c}0.96[0.01 ; 2.90] \\
35.8 \%\end{array}$ & NR & NR \\
\hline Hyperthyroidism & $\begin{array}{c}0.90[0.14 ; 2.16] \\
68.2 \%\end{array}$ & $\begin{array}{c}3.01[1.96 ; 4.24] \\
0.0 \%\end{array}$ & $\begin{array}{c}3.34[1.60 ; 5.61] \\
73.5 \%\end{array}$ & $\begin{array}{c}11.11[7.05 \\
17.07]\end{array}$ & $\begin{array}{c}10.16 \text { [5.94; } \\
15.28], 58.1 \%\end{array}$ & 0.00 [0.00; 10.43] & $N R$ \\
\hline Hypophysitis & $\begin{array}{c}4.13[2.35 ; 6.31] \\
74.5 \%\end{array}$ & $\begin{array}{c}0.31[0.00 ; 1.14] \\
0.0 \%\end{array}$ & $\begin{array}{c}0.66[0.05 ; 1.70] \\
61.2 \%\end{array}$ & $\begin{array}{c}10.46[6.54 ; \\
16.31]\end{array}$ & $\begin{array}{c}10.40 \text { [6.60; } \\
14.88], 56.9 \%\end{array}$ & $\begin{array}{c}3.76[0.61 ; 8.71] \\
0.0 \%\end{array}$ & $5.88[2.31 ; 14.17]$ \\
\hline Hypopituitarism & $\begin{array}{c}1.46[0.69 ; 2.45] \\
25.7 \%\end{array}$ & $\begin{array}{c}0.16[0.00 ; 0.88] \\
4.2 \%\end{array}$ & $\begin{array}{c}0.36[0.10 ; 0.75] \\
0.0 \%\end{array}$ & $0.65[0.12 ; 3.61]$ & $\begin{array}{c}1.25[0.18 ; 2.95] \\
0.0 \%\end{array}$ & NR & NR \\
\hline Hypothyroidism & $\begin{array}{c}2.84[1.46 ; 4.57] \\
64.8 \%\end{array}$ & $\begin{array}{c}7.02[4.37 ; 10.19] \\
60.3 \%\end{array}$ & $\begin{array}{c}8.34[7.01 ; 9.77] \\
0.0 \%\end{array}$ & $\begin{array}{c}16.34[11.32 ; \\
23.01]\end{array}$ & $\begin{array}{l}16.39 \text { [13.50; } \\
19.49], 1.7 \%\end{array}$ & $\begin{array}{c}11.22 \text { [0.03; } \\
33.98], 85.7 \%\end{array}$ & $\begin{array}{c}22.06[13.85 ; \\
\quad 33.26]\end{array}$ \\
\hline $\begin{array}{l}\text { Lymphocytic } \\
\text { hypophysitis }\end{array}$ & $\begin{array}{c}0.06[0.00 ; 0.39] \\
0.0 \%\end{array}$ & $0.00[0.00 ; 01.21]$ & $N R$ & 0.65 [0.12; 3.61] & $0.64[0.18 ; 2.30]$ & NR & $N R$ \\
\hline Thyroiditis & $\begin{array}{c}0.65[0.25 ; 1.20] \\
0.0 \%\end{array}$ & $\begin{array}{c}1.63[0.00 ; 4.96] \\
42.9 \%\end{array}$ & $\begin{array}{c}0.75[0.13 ; 1.80] \\
62.8 \%\end{array}$ & 5.23 [2.67; 9.98] & $\begin{array}{c}5.83[1.51 ; 12.30] \\
72.2 \%\end{array}$ & 0.00 [0.00; 10.43] & NR \\
\hline Thyrotoxic crisis & $0.14[0.02 ; 0.78]$ & NR & NR & NR & NR & NR & NR \\
\hline \multicolumn{8}{|l|}{ Eye disorders } \\
\hline Uveitis & $\begin{array}{c}0.93[0.03 ; 2.56] \\
73.2 \%\end{array}$ & $\begin{array}{c}0.24[0.00 ; 1.33] \\
0.0 \%\end{array}$ & $\begin{array}{c}0.70[0.28 ; 1.24] \\
0.0 \%\end{array}$ & 2.61 [1.02; 6.53] & $\begin{array}{c}2.03[0.19 ; 5.17] \\
55.3 \%\end{array}$ & 0.00 [0.00; 10.43] & $N R$ \\
\hline \multicolumn{8}{|c|}{ Gastrointestinal disorders } \\
\hline Autoimmune colitis & $\begin{array}{c}1.78[0.27 ; 4.20] \\
68.0 \%\end{array}$ & $0.64[0.18 ; 2.30]$ & $0.28[0.05 ; 1.57]$ & $1.96[0.67 ; 5.61]$ & $\begin{array}{c}1.16[0.10 ; 2.97] \\
38.6 \%\end{array}$ & $1.43[0.25 ; 7.66]$ & $0.00[0.00 ; 5.35]$ \\
\hline $\begin{array}{l}\text { Autoimmune } \\
\text { pancreatitis }\end{array}$ & $\begin{array}{c}0.00[0.00 ; 0.01] \\
0.0 \%\end{array}$ & 0.32 [0.06; 1.79] & 0.18 [0.03; 1.01] & 1.31 [0.36; 4.64] & $\begin{array}{c}0.17[0.00 ; 2.18] \\
62.6 \%\end{array}$ & $0.00[0.00 ; 5.20]$ & 0.00 [0.00; 5.35] \\
\hline Colitis & $\begin{array}{c}7.98[6.44 ; 9.66] \\
42.9 \%\end{array}$ & $\begin{array}{c}1.80[0.42 ; 3.86] \\
63.4 \%\end{array}$ & $\begin{array}{c}2.05[1.13 ; 3.20] \\
39.3 \%\end{array}$ & $9.15[5.53 ; 14.77]$ & $\begin{array}{c}13.35 \text { [9.52; } \\
17.68], 46.1 \%\end{array}$ & $\begin{array}{c}7.80[0.00 ; 42.08] \\
93.7 \%\end{array}$ & $\begin{array}{c}16.18 \text { [9.28; } \\
26.69]\end{array}$ \\
\hline Diarrhea & $\begin{array}{l}29.21[24.27 ; \\
34.40], 85.6 \%\end{array}$ & $\begin{array}{c}18.18 \text { [13.11; } \\
23.85], 76.2 \%\end{array}$ & $\begin{array}{c}14.09 \text { [10.54; } \\
18.02], 68.8 \%\end{array}$ & $\begin{array}{c}26.14 \text { [19.83; } \\
\text { 33.63] }\end{array}$ & $\begin{array}{l}40.46 \text { [34.70; } \\
46.36], 46.4 \%\end{array}$ & $\begin{array}{c}25.22 \text { [0.96; } \\
64.29 \text { ], 93.2\% }\end{array}$ & $\begin{array}{c}47.06[35.67 ; \\
58.76]\end{array}$ \\
\hline Enterocolitis & $\begin{array}{c}1.44[0.00 ; 4.68] \\
87.0 \%\end{array}$ & NR & $\begin{array}{c}0.00[0.00 ; 0.21] \\
0.0 \%\end{array}$ & NR & $\begin{array}{c}0.44[0.00 ; 1.39] \\
0.0 \%\end{array}$ & $1.43[0.25 ; 7.66]$ & 0.00 [0.00; 5.35] \\
\hline $\begin{array}{l}\text { Frequent bowel } \\
\text { movements }\end{array}$ & $0.39[0.07 ; 2.18]$ & $0.97[0.27 ; 3.47]$ & $0.54[0.18 ; 1.58]$ & $0.65[0.12 ; 3.61]$ & NR & NR & NR \\
\hline
\end{tabular}


TABLE 2 | Continued

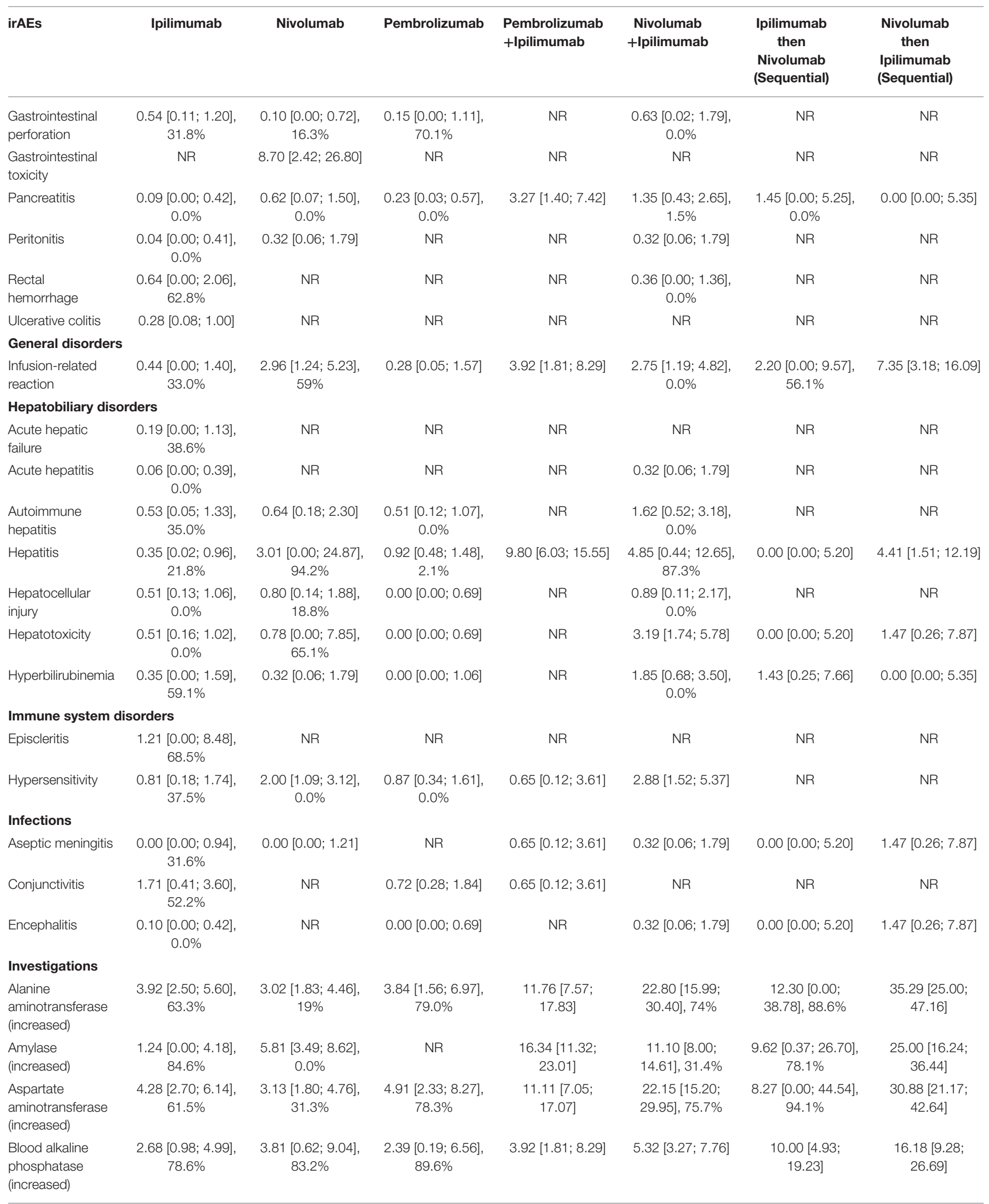


TABLE 2 | Continued

\begin{tabular}{|c|c|c|c|c|c|c|c|}
\hline irAEs & Ipilimumab & Nivolumab & Pembrolizumab & $\begin{array}{l}\text { Pembrolizumab } \\
\text { +Ipilimumab }\end{array}$ & $\begin{array}{l}\text { Nivolumab } \\
+ \text { Ipilimumab }\end{array}$ & $\begin{array}{c}\text { Ipilimumab } \\
\text { then } \\
\text { Nivolumab } \\
\text { (Sequential) }\end{array}$ & $\begin{array}{c}\text { Nivolumab } \\
\text { then } \\
\text { Ipilimumab } \\
\text { (Sequential) }\end{array}$ \\
\hline $\begin{array}{l}\text { Blood bilirubin } \\
\text { (increased) }\end{array}$ & $\begin{array}{c}0.65[0.00 ; 2.09] \\
69.9 \%\end{array}$ & $\begin{array}{c}0.27 \text { [0.00; 1.09], } \\
0.0 \%\end{array}$ & $\begin{array}{c}1.63[0.80 ; 2.73], \\
21.6 \%\end{array}$ & $0.65[0.12 ; 3.61]$ & $\begin{array}{c}2.55[0.86 ; 4.92], \\
34.6 \%\end{array}$ & $1.43[0.25 ; 7.66]$ & $\begin{array}{c}11.76[6.08 ; \\
21.53]\end{array}$ \\
\hline $\begin{array}{l}\text { Blood } \\
\text { corticotropin } \\
\text { (decreased) }\end{array}$ & $\begin{array}{c}0.47 \text { [0.00; 4.00], } \\
71.5 \%\end{array}$ & NR & $0.18[0.03 ; 1.01]$ & NR & NR & NR & NR \\
\hline $\begin{array}{l}\text { Blood creatinine } \\
\text { (increased) }\end{array}$ & $\begin{array}{c}0.60[0.02 ; 1.66] \\
63.9 \%\end{array}$ & $\begin{array}{c}0.89[0.15 ; 2.03] \\
32.2 \%\end{array}$ & $\begin{array}{c}0.99 \text { [0.00; 3.22] } \\
78.2 \%\end{array}$ & $2.61[1.02 ; 6.53]$ & $\begin{array}{c}3.44[1.94 ; 5.27] \\
0.0 \%\end{array}$ & $\begin{array}{c}0.00[0.00 ; 1.73] \\
0.0 \%\end{array}$ & $1.47[0.26 ; 7.87]$ \\
\hline $\begin{array}{l}\text { Blood thyroid } \\
\text { stimulating } \\
\text { hormone } \\
\text { (abnormal) }\end{array}$ & $\begin{array}{c}1.68[0.03 ; 4.83] \\
57.2 \%\end{array}$ & $\begin{array}{c}2.75[0.01 ; 8.82], \\
81.8 \%\end{array}$ & $\begin{array}{c}2.34 \text { [1.12; 3.95], } \\
24.6 \%\end{array}$ & $3.27[1.40 ; 7.42]$ & $\begin{array}{c}6.66[0.04 ; 20.47], \\
82.8 \%\end{array}$ & $\begin{array}{c}2.32[0.00 ; 10.59] \\
61.0 \%\end{array}$ & $2.94[0.81 ; 10.10]$ \\
\hline $\begin{array}{l}\text { Blood urea } \\
\text { (increased) }\end{array}$ & NR & NR & NR & NR & $1.06[0.19 ; 5.78]$ & NR & NR \\
\hline $\begin{array}{l}\text { Gamma-glutamyl } \\
\text { transferase } \\
\text { (increased) }\end{array}$ & $\begin{array}{c}0.75[0.27 ; 1.38] \\
0.0 \%\end{array}$ & $\begin{array}{c}0.55[0.00 ; 3.93] \\
52.1 \%\end{array}$ & NR & $7.19[4.06 ; 12.41]$ & $\begin{array}{c}3.29[1.68 ; 5.30] \\
0.0 \%\end{array}$ & $0.00[0.00 ; 10.43]$ & NR \\
\hline $\begin{array}{l}\text { Hepatic enzyme } \\
\text { (increased) }\end{array}$ & $\begin{array}{c}0.19 \text { [0.00; 0.75] } \\
31.6 \%\end{array}$ & $\begin{array}{c}0.34[0.00 ; 1.07] \\
0.0 \%\end{array}$ & $0.28[0.05 ; 1.57]$ & $0.65[0.12 ; 3.61]$ & $1.60[0.68 ; 3.68]$ & $0.00[0.00 ; 10.43]$ & $1.47[0.26 ; 7.87]$ \\
\hline Lipase (increased) & $\begin{array}{c}2.75[0.56 ; 6.12] \\
86.2 \%\end{array}$ & $\begin{array}{c}3.10[0.00 ; 11.74] \\
85.4 \%\end{array}$ & NR & $\begin{array}{c}20.92[15.22 \\
28.03]\end{array}$ & $\begin{array}{c}15.78[12.50 \\
19.35], 19.2 \%\end{array}$ & $\begin{array}{c}21.25[6.77 \\
40.55], 75.2 \%\end{array}$ & $\begin{array}{c}32.35[22.44 \\
44.16]\end{array}$ \\
\hline $\begin{array}{l}\text { Liver function test } \\
\text { (abnormal) }\end{array}$ & $\begin{array}{c}0.00[0.00 ; 0.23] \\
0.0 \%\end{array}$ & $\begin{array}{c}0.64[0.07 ; 1.63] \\
17.8 \%\end{array}$ & $0.18[0.03 ; 1.01]$ & $0.65[0.12 ; 3.61]$ & $\begin{array}{c}0.36[0.00 ; 1.36] \\
0.0 \%\end{array}$ & $N R$ & NR \\
\hline $\begin{array}{l}\text { Thyroxine free } \\
\text { (decreased) }\end{array}$ & NR & $1.87[0.51 ; 6.56]$ & $2.31[0.90 ; 5.79]$ & $1.31[0.36 ; 4.64]$ & NR & NR & NR \\
\hline $\begin{array}{l}\text { Thyroxine } \\
\text { (increased) }\end{array}$ & NR & NR & $0.58[0.10 ; 3.20]$ & NR & NR & NR & NR \\
\hline $\begin{array}{l}\text { Transaminases } \\
\text { (increased) }\end{array}$ & $\begin{array}{c}0.68[0.22 ; 1.32] \\
0.0 \%\end{array}$ & $0.64[0.18 ; 2.30]$ & $\begin{array}{c}1.29[0.00 ; 4.39], \\
3.8 \%\end{array}$ & $0.65[0.12 ; 3.61]$ & $\begin{array}{c}2.48[1.29 ; 3.98] \\
0.0 \%\end{array}$ & NR & NR \\
\hline \multicolumn{8}{|c|}{ Metabolism and nutrition disorders } \\
\hline Diabetes mellitus & $\begin{array}{c}0.07[0.00 ; 4.52] \\
57.7 \%\end{array}$ & $\begin{array}{c}0.44[0.00 ; 1.35] \\
0.0 \%\end{array}$ & $\begin{array}{c}0.09 \text { [0.00; 0.69], } \\
37.5 \%\end{array}$ & $0.65[0.12 ; 3.61]$ & $0.96[0.33 ; 2.78]$ & $N R$ & NR \\
\hline $\begin{array}{l}\text { Diabetic } \\
\text { ketoacidosis }\end{array}$ & $\begin{array}{c}0.00 \text { [0.00; } 0.36] \\
35.8 \%\end{array}$ & $\begin{array}{c}0.11 \text { [0.00; } 0.94] \\
35.4 \%\end{array}$ & $0.18[0.03 ; 1.01]$ & $0.65[0.12 ; 3.61]$ & $\begin{array}{c}0.36[0.00 ; 1.36] \\
0.0 \%\end{array}$ & NR & NR \\
\hline Hyperglycemia & $\begin{array}{c}4.42[0.62 ; 10.65] \\
91.8 \%\end{array}$ & $\begin{array}{c}0.58[0.10 ; 1.31] \\
0.0 \%\end{array}$ & $\begin{array}{c}5.86[3.65 ; 8.50], \\
0.0 \%\end{array}$ & $1.31[0.36 ; 4.64]$ & $\begin{array}{c}2.60[1.19 ; 4.46] \\
0.0 \%\end{array}$ & $2.86[0.79 ; 9.83]$ & 7.35 [3.18; 16.09] \\
\hline Hyperlipasemia & $0.14[0.02 ; 0.78]$ & NR & NR & NR & NR & NR & NR \\
\hline $\begin{array}{l}\text { Type } 1 \text { diabetes } \\
\text { mellitus }\end{array}$ & $0.00[0.00 ; 1.48]$ & NR & $\begin{array}{c}0.09 \text { [0.00; 0.69], } \\
37.5 \%\end{array}$ & $1.96[0.67 ; 5.61]$ & $1.06[0.19 ; 5.78]$ & NR & NR \\
\hline \multicolumn{8}{|c|}{ Musculoskeletal and connective tissue disorders } \\
\hline Arthralgia & $\begin{array}{c}6.32[3.64 ; 9.60], \\
84.6 \%\end{array}$ & $\begin{array}{c}9.13[5.81 ; 13.04] \\
68 \%\end{array}$ & $\begin{array}{c}12.16[8.54 ; \\
16.29], 74.1 \%\end{array}$ & $\begin{array}{c}13.07[8.62 ; \\
19.33]\end{array}$ & $\begin{array}{l}14.82[12.10 \\
17.77], 0.0 \%\end{array}$ & $\begin{array}{c}9.60[4.40 ; 16.31] \\
0.0 \%\end{array}$ & $\begin{array}{c}22.06[13.85 \\
33.26]\end{array}$ \\
\hline Arthritis & $\begin{array}{c}0.20[0.00 ; 1.39] \\
65.7 \%\end{array}$ & $\begin{array}{c}0.10 \text { [0.00; } 0.72] \\
16.3 \%\end{array}$ & $\begin{array}{c}1.21[0.19 ; 2.83] \\
48.3 \%\end{array}$ & $0.65[0.12 ; 3.61]$ & $0.32[0.06 ; 1.79]$ & $0.00[0.00 ; 5.20]$ & $5.88[2.31 ; 14.17]$ \\
\hline Arthropathy & $0.32[0.06 ; 1.80]$ & $0.32[0.06 ; 1.79]$ & $0.58[0.10 ; 3.20]$ & NR & $0.64[0.18 ; 2.30]$ & $\mathrm{NR}$ & NR \\
\hline $\begin{array}{l}\text { Hand-foot- } \\
\text { syndrome } \\
\text { (Palmar-plantar } \\
\text { erythrodysesthesia } \\
\text { syndrome) }\end{array}$ & $0.97[0.17 ; 5.30]$ & NR & NR & NR & NR & NR & $N R$ \\
\hline Joint swelling & NR & NR & 0.58 [0.10; 3.20] & $0.65[0.12 ; 3.61]$ & NR & NR & NR \\
\hline Muscle spasms & $\begin{array}{c}0.51 \text { [0.02; 1.39] } \\
18.1 \%\end{array}$ & $\begin{array}{c}3.35[0.84 ; 7.32], \\
78.7 \%\end{array}$ & $\begin{array}{c}0.59[0.01 ; 1.73] \\
64.4 \%\end{array}$ & $1.96[0.67 ; 5.61]$ & $0.64[0.18 ; 2.30]$ & $0.00[0.00 ; 5.20]$ & $2.94[0.81 ; 10.10]$ \\
\hline
\end{tabular}


TABLE 2 | Continued

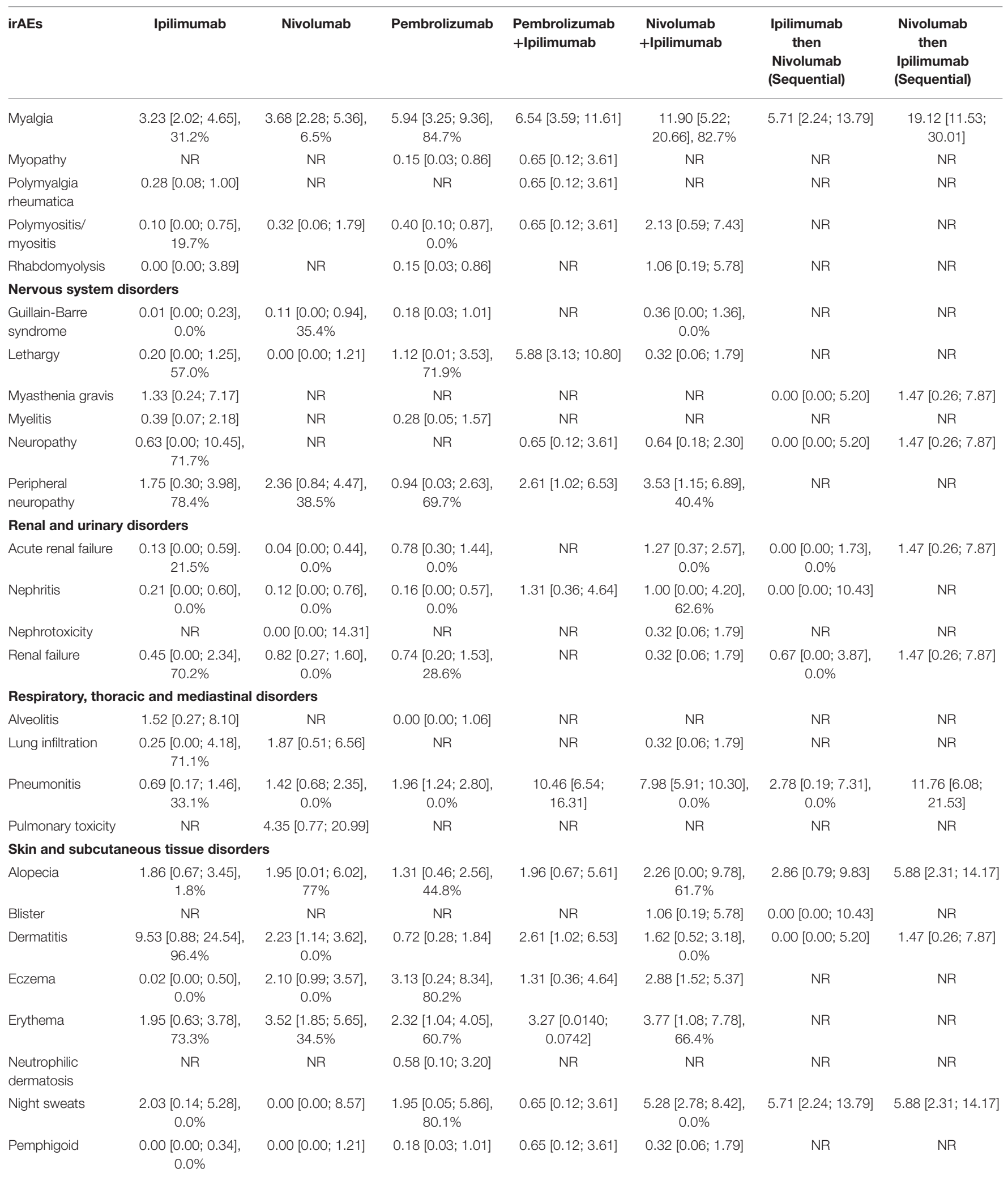


TABLE 2 | Continued

\begin{tabular}{|c|c|c|c|c|c|c|c|}
\hline irAEs & Ipilimumab & Nivolumab & Pembrolizumab & $\begin{array}{l}\text { Pembrolizumab } \\
\text { +Ipilimumab }\end{array}$ & $\begin{array}{l}\text { Nivolumab } \\
+ \text { Ipilimumab }\end{array}$ & $\begin{array}{c}\text { Ipilimumab } \\
\text { then } \\
\text { Nivolumab } \\
\text { (Sequential) }\end{array}$ & $\begin{array}{c}\text { Nivolumab } \\
\text { then } \\
\text { Ipilimumab } \\
\text { (Sequential) }\end{array}$ \\
\hline $\begin{array}{l}\text { Photosensitivity } \\
\text { reaction }\end{array}$ & NR & $\begin{array}{c}2.22[0.46 ; 5.00] \\
37.5 \%\end{array}$ & NR & 0.65 [0.12; 3.61] & $N R$ & $N R$ & $N R$ \\
\hline Pruritus & $\begin{array}{l}26.78 \text { [21.65; } \\
32.23], 86.8 \%\end{array}$ & $\begin{array}{l}21.43 \text { [13.93; } \\
29.99], 87.9 \%\end{array}$ & $\begin{array}{l}22.36 \text { [18.77; } \\
26.16], 56.3 \%\end{array}$ & $\begin{array}{c}41.18 \text { [33.69; } \\
49.10]\end{array}$ & $\begin{array}{l}39.06 \text { [31.68; } \\
46.69], 67.8 \%\end{array}$ & $\begin{array}{l}30.62 \text { [9.91; } \\
56.36], 84 \%\end{array}$ & $\begin{array}{c}35.29 \text { [25.00; } \\
\quad 47.16]\end{array}$ \\
\hline Rash & $\begin{array}{l}31.07 \text { [24.37; } \\
38.17], 90.9 \%\end{array}$ & $\begin{array}{c}19.62 \text { [12.65; } \\
27.65], 86.8 \%\end{array}$ & $\begin{array}{l}17.14 \text { [11.69; } \\
23.35], 85.4 \%\end{array}$ & $\begin{array}{c}41.83 \text { [34.31; } \\
49.75]\end{array}$ & $\begin{array}{l}39.76 \text { [20.91; } \\
60.31], 95.7 \%\end{array}$ & $\begin{array}{c}20.38 \text { [2.81; } \\
47.13], 86.6 \%\end{array}$ & $\begin{array}{c}39.71[28.93 \\
51.58]\end{array}$ \\
\hline $\begin{array}{l}\text { Rash } \\
\text { erythematous }\end{array}$ & $\begin{array}{c}0.92[0.00 ; 3.01] \\
55.8 \%\end{array}$ & $1.12[0.38 ; 3.24]$ & $0.72[0.28 ; 1.84]$ & $1.96[0.67 ; 5.61]$ & $1.06[0.19 ; 5.78]$ & NR & NR \\
\hline Rash generalized & $\begin{array}{c}2.71[0.03 ; 7.97] \\
75.0 \%\end{array}$ & $\begin{array}{c}0.88[0.04 ; 2.42] \\
21.5 \%\end{array}$ & 1.73 [0.59; 4.97] & 1.31 [0.36; 4.64] & $\begin{array}{c}2.37[1.02 ; 4.16] \\
0.0 \%\end{array}$ & NR & NR \\
\hline Rash macular & 0.32 [0.06; 1.80] & $\begin{array}{c}0.88[0.04 ; 2.42] \\
21.5 \%\end{array}$ & NR & 4.58 [2.23; 9.14] & $\begin{array}{c}2.47[1.20 ; 4.10] \\
0.0 \%\end{array}$ & $N R$ & NR \\
\hline $\begin{array}{l}\text { Rash } \\
\text { maculo-papular }\end{array}$ & $\begin{array}{c}5.47[1.60 ; 11.24] \\
92.8 \%\end{array}$ & $\begin{array}{c}13.14 \text { [2.61; } \\
29.39], 97.2 \%\end{array}$ & $\begin{array}{c}3.26[1.13 ; 6.30] \\
80.1 \%\end{array}$ & $\begin{array}{c}12.42[8.10 \\
18.58]\end{array}$ & $\begin{array}{c}13.84 \text { [3.73; } \\
28.67], 94.3 \%\end{array}$ & $\begin{array}{c}17.14 \text { [10.09; } \\
27.62]\end{array}$ & $\begin{array}{c}23.53[15.03 \\
\quad 34.86]\end{array}$ \\
\hline Rash papular & $\begin{array}{c}1.15[0.47 ; 2.07] \\
0.0 \%\end{array}$ & $\begin{array}{c}1.68[0.70 ; 3.03] \\
0.0 \%\end{array}$ & $\begin{array}{c}0.36[0.01 ; 1.01] \\
0.0 \%\end{array}$ & 0.65 [0.12; 3.61] & $\begin{array}{c}3.19[0.79 ; 6.91] \\
55.2 \%\end{array}$ & NR & NR \\
\hline Rash pruritic & $\begin{array}{c}2.33[1.21 ; 3.75] \\
53.4 \%\end{array}$ & $\begin{array}{c}1.09[0.00 ; 4.78] \\
75.3 \%\end{array}$ & $1.26[0.61 ; 2.58]$ & $5.88[3.13 ; 10.80]$ & $\begin{array}{c}2.02[0.87 ; 3.54] \\
0.0 \%\end{array}$ & $\begin{array}{c}10.00 \text { [4.93; } \\
19.23]\end{array}$ & $1.47[0.26 ; 7.87]$ \\
\hline Skin exfoliation & 5.00 [0.89; 23.61] & $1.87[0.51 ; 6.56]$ & NR & 1.96 [0.67; 5.61] & NR & NR & NR \\
\hline $\begin{array}{l}\text { Skin } \\
\text { hypopigmentation }\end{array}$ & $\begin{array}{c}0.23[0.00 ; 1.55] \\
51.6 \%\end{array}$ & $2.24[1.09 ; 4.54]$ & $\begin{array}{c}1.35[0.49 ; 2.53] \\
7.3 \%\end{array}$ & NR & $\begin{array}{c}3.51[0.34 ; 9.23] \\
76.2 \%\end{array}$ & NR & $N R$ \\
\hline Skin reactions & $N R$ & $N R$ & NR & 8.50 [5.03; 13.99] & NR & $N R$ & NR \\
\hline Toxic skin eruption & $\begin{array}{c}0.00[0.00 ; 0.31] \\
35.3 \%\end{array}$ & $0.32[0.06 ; 1.79]$ & $2.38[0.42 ; 12.32]$ & 3.92 [1.81; 8.29] & NR & NR & NR \\
\hline Urticaria & $\begin{array}{c}0.96[0.02 ; 2.76] \\
33.2 \%\end{array}$ & $1.87[0.51 ; 6.56]$ & $9.52[3.77 ; 22.07]$ & $0.65[0.12 ; 3.61]$ & $2.13[0.59 ; 7.43]$ & 0.00 [0.00; 10.43] & NR \\
\hline Vitiligo & $\begin{array}{c}2.67[1.05 ; 4.82] \\
67.9 \%\end{array}$ & $\begin{array}{c}9.05[5.41 ; 13.42] \\
72.9 \%\end{array}$ & $\begin{array}{c}9.40[7.99 ; 10.90] \\
0.0 \%\end{array}$ & $\begin{array}{c}19.61[14.09 \\
26.61]\end{array}$ & $\begin{array}{c}9.96[6.80 ; 13.60] \\
39.8 \%\end{array}$ & $\begin{array}{c}6.41[0.00 ; 34.52] \\
91.7 \%\end{array}$ & $\begin{array}{c}14.71[8.19 ; \\
25.00]\end{array}$ \\
\hline \multicolumn{8}{|l|}{ Vascular disorders } \\
\hline $\begin{array}{l}\text { Thromboembolic } \\
\text { event }\end{array}$ & $\begin{array}{c}0.36[0.00 ; 1.29] \\
54.4 \%\end{array}$ & $\begin{array}{c}0.49[0.07 ; 1.17] \\
0.0 \%\end{array}$ & $\begin{array}{c}0.64[0.19 ; 1.31] \\
0.0 \%\end{array}$ & $N R$ & $\begin{array}{c}0.79[0.00 ; 3.46] \\
61.0 \%\end{array}$ & $4.29[1.47 ; 11.86]$ & $1.47[0.26 ; 7.87]$ \\
\hline
\end{tabular}

$N R$, not reported.

$1.5 \%$, respectively), all-grade elevated blood alkaline phosphatase (3.8\%), and all-grade elevated blood creatinine (1.1\%). The incidence of all-grade elevated blood bilirubin, AST, ALT, and abnormal TSH was high among pembrolizumab users.

\section{Metabolism and Nutritional Disorders}

Type 1 diabetes mellitus (all-grade and grade $\geq 3$ ) showed an incidence of $2.0 \%$ after the combination of ipilimumab and pembrolizumab and an incidence of $1.0 \%$ after the combination of ipilimumab and nivolumab. The incidence of all-grade hyperglycemia was $7.4 \%$ after sequential nivolumab followed by ipilimumab and that of grade $\geq 3$ hyperglycemia was $2.9 \%$ after sequential ipilimumab followed by nivolumab. After the FDAapproved dose of pembrolizumab, the incidence of both all-grade and grade $\geq 3$ type 1 diabetes mellitus was $2.4 \%$ and that of all-grade hyperglycemia was $5.6 \%$. The incidence of all-grade and grade $\geq 3$ hyperglycemia was 2.6 and $1.6 \%$, respectively, after the FDA-approved doses of the combination of nivolumab and ipilimumab.

\section{Musculoskeletal and Connective Tissue Disorders}

The most common musculoskeletal and connective disorders, i.e., potential irAEs, were arthralgia and myalgia. They were reported frequently after combination therapies. Among monotherapies, pembrolizumab users had the most frequent incidence of all-grade arthralgia (12.2\%), all-grade myalgia (5.9\%), and all-grade arthritis (1.2\%). Moreover, the incidence of all-grade muscle spasm was $3.4 \%$ after nivolumab. The most frequent potential irAEs among ipilimumab monotherapy users were all-grade arthralgia (6.3\%) and all-grade myalgia (3.2\%). Regarding the FDA-approved doses of ICIs, the combination of nivolumab and ipilimumab resulted in the highest incidence of all-grade arthralgia (14.6\%), all-grade myalgia (11.9\%), all-grade muscle spasm $(2.2 \%)$, all-grade polymyositis $(2.1 \%)$, and allgrade rhabdomyolysis (1.1\%). After the FDA-approved doses of monotherapies, the incidence of all-grade arthralgia and myalgia was, respectively, 6.2 and $3.2 \%$ for ipilimumab; 9.4 and $4.2 \%$ for nivolumab; and 7.7 and $4.9 \%$ for pembrolizumab. All-grade muscle spasm was reported in $3.4 \%$ of nivolumab users. 


\section{Nervous System Disorders}

The combination of nivolumab and ipilimumab resulted in an incidence of $3.5 \%$ for all-grade peripheral neuropathy. The incidence of all-grade myasthenia gravis and neuropathy was $1.5 \%$ after sequential nivolumab followed by ipilimumab. After pembrolizumab and ipilimumab combination therapy, the incidence of all-grade lethargy was $5.9 \%$ and that of all-grade peripheral neuropathy was $2.6 \%$. Among pembrolizumab users, the incidence of all-grade lethargy was $1.1 \%$. The incidence of all-grade peripheral neuropathy was $2.4 \%$ after nivolumab monotherapy. The incidence of irAEs was similar after FDAapproved doses of the combination of nivolumab and ipilimumab as well as nivolumab and pembrolizumab therapies, as described above. Moreover, the incidence of myasthenia gravis was $1.3 \%$ after ipilimumab.

\section{Renal and Urinary Disorders}

The incidence of all-grade and grade $\geq 3$ acute renal failure was 1.3 and $1.1 \%$, respectively, after combination nivolumab and ipilimumab. Sequential nivolumab followed by ipilimumab resulted in an incidence of $1.5 \%$ for all-grade and grade $\geq 3$ acute renal failure. All-grade nephritis was observed in $1.3 \%$ of patients receiving the combination of pembrolizumab and ipilimumab and in $1 \%$ of those receiving nivolumab and ipilimumab combination therapy. After ICI monotherapies, the frequency of renal and urinary disorders with potential irAEs was $<1 \%$. The incidence of potential irAEs related to the renal system was also $<1 \%$ after FDA-approved doses of ICI combination or monotherapies.

\section{Respiratory, Thoracic, and Mediastinal Disorders}

Pneumonitis (all-grade and grade $\geq 3$ ) was the most common potential respiratory irAE reported in the included trials, especially after combination therapies. The incidence of allgrade pneumonitis was $2.0 \%$ after pembrolizumab and $1.4 \%$ after nivolumab monotherapies. After ipilimumab, the incidence of all-grade alveolitis was $15 \%$, while after nivolumab, the incidence of all-grade pulmonary toxicity was $4.4 \%$. After the FDAapproved dose of ICIs, the incidence of all-grade pneumonitis was $7.5 \%$ after combination nivolumab and ipilimumab, $1.5 \%$ after pembrolizumab, and $1.4 \%$ after nivolumab.

\section{Skin and Subcutaneous Tissue Disorders}

Combination therapies resulted in frequent potential skin irAEs, especially all-grade alopecia, pruritus, rash, macular rash, maculopapular rash, toxic skin eruptions, vitiligo, and grade $\geq 3$ pruritus and rash. After nivolumab and ipilimumab combination therapy, the incidence of grade $\geq 3$ rash was $3.6 \%$ and that of maculopapular rash was $1.4 \%$. After ipilimumab, all-grade rash was observed in $31.1 \%$ of patients, pruritus in $26.8 \%$, dermatitis in $9.5 \%$, skin exfoliation in $5.0 \%$, generalized rash in $2.7 \%$, pruritic rash in $2.3 \%$, and night sweats in $2.0 \%$. After pembrolizumab, the incidence of all-grade eczema was $3.1 \%$, that of toxic skin eruptions was $2.4 \%$, that of urticaria was $9.5 \%$, and that of vitiligo was $9.4 \%$. After nivolumab, allgrade alopecia was observed in $2.0 \%$ of patients, erythema in $3.5 \%$, photosensitivity reaction in $2.2 \%$, erythematous rash in $1.1 \%$, maculopapular rash in $13.1 \%$, papular rash in $1.7 \%$, and skin hypopigmentation in $2.2 \%$. After the FDA approved doses of the combination of nivolumab and ipilimumab, the incidence of all-grade pruritus and rash and grade $\geq 3$ rash was $33.7,30.7$, and $3.0 \%$, respectively. The most frequent potential skin-related irAEs after the FDA-approved dose of ipilimumab included all-grade rash $(20.5 \%)$, pruritus $(24.9 \%)$, dermatitis (4.7\%), generalized rash $(1.3 \%)$, pruritic rash $(2.4 \%)$, and night sweats $(2.0 \%)$. The FDA-approved dose of pembrolizumab was associated with all-grade alopecia (3.4\%), eczema (4.5\%), erythema $(4.7 \%)$, toxic skin eruptions $(2.4 \%)$, and urticaria (9.5\%). The FDA-approved dose of nivolumab resulted in all-grade photosensitivity reaction (3.8\%), maculopapular rash (16.1\%), papular rash $(1.7 \%)$, skin hypopigmentation $(2.2 \%)$, and vitiligo (9.1\%).

\section{Vascular Disorders}

The incidence of all-grade and grade $\geq 3$ thromboembolic events was most frequent after the combination therapies. After monotherapies, the incidence of all-grade and grade $\geq 3$ thromboembolic events was $<1 \%$; however, the FDA-approved dose of pembrolizumab resulted in an incidence of $1.1 \%$ for all-grade thromboembolic events.

\section{Heterogeneity}

A high heterogeneity $\left(I^{2}>75 \%\right)$ was observed in 60 outcomes (Tables 2 and 3). Restricting the doses to the FDA-approved doses of ICIs decreased the heterogeneity in 21 outcomes to $I^{2}<75 \%$ (Tables 4 and 5). Subgroup analyses were not feasible because the data were presented from two trials only in 10 outcomes of sequential ipilimumab followed by nivolumab (Figures S17-S26), 4 outcomes of nivolumab monotherapy (Figures S27-S30), and 1 outcome of ipilimumab and nivolumab combination therapy (Figure S31). For the remaining outcomes, subgroup analysis based on the prior systemic anticancer therapy use showed a reduction in the heterogeneity in most studies that included previously untreated patients (Figures S32-S55). There were 7 more outcomes in the FDA-approved doses of ICIs with high heterogeneity (Tables 4 and 5). Four outcomes were based on 2 studies, and subgrouping was not applicable (Figures S56S59). In the remaining 3 outcomes, subgrouping based on prior systemic anticancer therapy showed a low heterogeneity in previously untreated patients (Figures S60-S62).

\section{DISCUSSION}

This systematic review and meta-analysis summarized the final evidence on irAEs reported in clinical trials of monoand combination therapy of ipilimumab, nivolumab, and pembrolizumab in the treatment of advanced melanoma, and profiled the potential irAEs rates across these trials. The beneficial effects of using a combination of anti-CTLA4 or anti-PD-1 agents were associated with an elevated incidence of potential irAEs. Anti-CTLA-4 therapy was mostly associated with an elevated incidence of potential irAEs of the gastrointestinal tract (colitis, diarrhea, enterocolitis, and gastrointestinal perforation), renal system (nephritis), skin 
TABLE 3 | Incidence of Grades $\geq 3$ Potential Immune-Related Adverse Events (irAEs) in Advanced Melanoma presented as $\%$ with $95 \%$ confidence interval, and $\ell^{2}$ for the $\%$ of heterogeneity ( $l^{2}$ not reported if the outcome was obtained from one study).

\begin{tabular}{|c|c|c|c|c|c|c|c|}
\hline irAEs & Ipilimumab & Nivolumab & Pembrolizumab & $\begin{array}{l}\text { Pembrolizumab } \\
\text { +Ipilimumab }\end{array}$ & $\begin{array}{l}\text { Nivolumab } \\
\text { +Ipilimumab }\end{array}$ & $\begin{array}{c}\text { Ipilimumab } \\
\text { then } \\
\text { Nivolumab } \\
\text { (Sequential) }\end{array}$ & $\begin{array}{c}\text { Nivolumab } \\
\text { then } \\
\text { Ipilimumab } \\
\text { (Sequential) }\end{array}$ \\
\hline Number of studies & 21 & 7 & 5 & 1 & 5 & 2 & 1 \\
\hline Overall incidence & $\begin{array}{l}19.50 \text { [14.52; } \\
24.99], 90.0 \%\end{array}$ & $\begin{array}{c}4.00[1.00 ; 6.00] \\
66.0 \%\end{array}$ & $\begin{array}{c}4.78[2.65 ; 7.41] \\
69.0 \%\end{array}$ & $\begin{array}{c}27.45[21.00 \\
35.01]\end{array}$ & $\begin{array}{l}36.00 \text { [21.00; } \\
52.00], 93.0 \%\end{array}$ & $\begin{array}{c}13.00 \text { [3.00; } \\
29.00], 71.2 \%\end{array}$ & $\begin{array}{c}15.00[8.00 ; \\
25.00]\end{array}$ \\
\hline \multicolumn{8}{|c|}{ Blood and lymphatic system disorders } \\
\hline Lymphopenia & $\begin{array}{c}0.00[0.00 ; 3.89] \\
0.0 \%\end{array}$ & $\begin{array}{c}0.76[0.00 ; 5.65] \\
83.4 \%\end{array}$ & $0.00[0.00 ; 8.38]$ & NR & NR & NR & NR \\
\hline \multicolumn{8}{|l|}{ Cardiac disorders } \\
\hline Pericarditis & $0.14[0.02 ; 0.78]$ & $0.37[0.07 ; 2.08]$ & $0.15[0.03 ; 0.86]$ & NR & NR & NR & NR \\
\hline $\begin{array}{l}\text { Ventricular } \\
\text { arrhythmia }\end{array}$ & $0.00[0.00 ; 7.71]$ & $0.37[0.07 ; 2.08]$ & NR & NR & $0.00[0.00 ; 3.93]$ & NR & $N R$ \\
\hline \multicolumn{8}{|l|}{ Endocrine disorders } \\
\hline $\begin{array}{l}\text { Acute } \\
\text { adrenocortical } \\
\text { insufficiency }\end{array}$ & $\begin{array}{c}0.14[0.00 ; 0.53] \\
0.0 \%\end{array}$ & $0.00[0.00 ; 1.21]$ & $0.28[0.05 ; 1.57]$ & $0.65[0.12 ; 3.61]$ & $0.32[0.06 ; 1.79]$ & NR & NR \\
\hline $\begin{array}{l}\text { Adrenal } \\
\text { insufficiency }\end{array}$ & $\begin{array}{c}0.06[0.00 ; 0.41] \\
11.7 \%\end{array}$ & $\begin{array}{c}0.77[0.07 ; 1.97] \\
25.2 \%\end{array}$ & $\begin{array}{c}0.33[0.03 ; 0.82] \\
0.0 \%\end{array}$ & $0.65[0.12 ; 3.61]$ & $\begin{array}{c}1.57[0.63 ; 2.84] \\
0.0 \%\end{array}$ & $\begin{array}{c}0.58 \text { [0.00; 5.93] } \\
50.5 \%\end{array}$ & $4.41[1.51 ; 12.19]$ \\
\hline $\begin{array}{l}\text { Adrenocorticotropic } \\
\text { hormone } \\
\text { deficiency }\end{array}$ & $0.14[0.02 ; 0.78]$ & NR & NR & $0.00[0.00 ; 2.45]$ & NR & NR & NR \\
\hline $\begin{array}{l}\text { Autoimmune } \\
\text { thyroiditis }\end{array}$ & $\begin{array}{c}0.00[0.00 ; 0.16] \\
0.0 \%\end{array}$ & $0.00[0.00 ; 1.21]$ & $N R$ & NR & $\begin{array}{c}0.36[0.00 ; 1.36] \\
0.0 \%\end{array}$ & NR & NR \\
\hline Hyperthyroidism & $\begin{array}{c}0.10 \text { [0.00; 0.43] } \\
1.0 \%\end{array}$ & $\begin{array}{c}0.00[0.00 ; 0.09] \\
0.0 \%\end{array}$ & $\begin{array}{c}0.00[0.00 ; 0.13] \\
0.0 \%\end{array}$ & $1.31[0.36 ; 4.64]$ & $\begin{array}{c}0.66[0.00 ; 2.08] \\
25.1 \%\end{array}$ & $0.00[0.00 ; 10.43]$ & NR \\
\hline Hypophysitis & $\begin{array}{c}2.06[0.87 ; 3.60] \\
69.0 \%\end{array}$ & $\begin{array}{c}0.15[0.00 ; 0.87] \\
0.0 \%\end{array}$ & $\begin{array}{c}0.15 \text { [0.00; 0.58] } \\
19.8 \%\end{array}$ & $1.96[0.67 ; 5.61]$ & $\begin{array}{c}2.36[1.01 ; 4.14] \\
20.6 \%\end{array}$ & $\begin{array}{c}2.78[0.19 ; 7.31] \\
0.0 \%\end{array}$ & $0.00[0.00 ; 5.35]$ \\
\hline Hypopituitarism & $\begin{array}{c}0.96[0.27 ; 1.95] \\
38.8 \%\end{array}$ & $\begin{array}{c}0.08[0.00 ; 0.71] \\
0.0 \%\end{array}$ & $\begin{array}{c}0.14 \text { [0.00; 0.59] } \\
48.4 \%\end{array}$ & $0.00[0.00 ; 2.45]$ & $\begin{array}{c}0.43[0.00 ; 1.71] \\
0.0 \%\end{array}$ & NR & NR \\
\hline Hypothyroidism & $\begin{array}{c}0.00 \text { [0.00; } 0.05] \\
0.0 \%\end{array}$ & $\begin{array}{c}0.00[0.00 ; 0.07] \\
0.0 \%\end{array}$ & $\begin{array}{c}0.00[0.00 ; 0.18] \\
0.0 \%\end{array}$ & $0.00[0.00 ; 2.45]$ & $\begin{array}{c}0.08[0.00 ; 0.69] \\
0.0 \%\end{array}$ & $\begin{array}{c}0.00[0.00 ; 1.73] \\
0.0 \%\end{array}$ & $0.00[0.00 ; 5.35]$ \\
\hline $\begin{array}{l}\text { Lymphocytic } \\
\text { hypophysitis }\end{array}$ & $\begin{array}{c}0.01[0.00 ; 0.27] \\
0.0 \%\end{array}$ & $0.00[0.00 ; 1.21]$ & NR & $0.00[0.00 ; 2.45]$ & $0.32[0.06 ; 1.79]$ & NR & NR \\
\hline Thyroiditis & $\begin{array}{c}0.02[0.00 ; 0.25] \\
0.0 \%\end{array}$ & $\begin{array}{c}0.00[0.00 ; 0.15] \\
0 \%\end{array}$ & $\begin{array}{c}0.00[0.00 ; 0.16] \\
0.0 \%\end{array}$ & $0.00[0.00 ; 2.45]$ & $\begin{array}{c}0.02[0.00 ; 0.67] \\
0.0 \%\end{array}$ & $0.00[0.00 ; 10.43]$ & NR \\
\hline Thyrotoxic crisis & $0.14[0.02 ; 0.78]$ & NR & NR & NR & NR & NR & NR \\
\hline \multicolumn{8}{|l|}{ Eye disorders } \\
\hline Uveitis & $\begin{array}{c}0.09[0.00 ; 0.79] \\
47.1 \%\end{array}$ & $\begin{array}{c}0.00[0.00 ; 0.22] \\
0.0 \%\end{array}$ & $\begin{array}{c}0.00 \text { [0.00; } 0.05] \\
0.0 \%\end{array}$ & 0.00 [0.00; 2.45] & $\begin{array}{c}0.42[0.00 ; 2.79] \\
61.9 \%\end{array}$ & 0.00 [0.00; 10.43] & $N R$ \\
\hline \multicolumn{8}{|c|}{ Gastrointestinal disorders } \\
\hline Autoimmune colitis & $\begin{array}{c}0.71[0.17 ; 1.49] \\
11.7 \%\end{array}$ & $0.32[0.06 ; 1.79]$ & $0.28[0.05 ; 1.57]$ & $1.96[0.67 ; 5.61]$ & $\begin{array}{c}1.04 \text { [0.00; 3.33] } \\
57.7 \%\end{array}$ & $1.43[0.25 ; 7.66]$ & $0.00[0.00 ; 5.35]$ \\
\hline $\begin{array}{l}\text { Autoimmune } \\
\text { pancreatitis }\end{array}$ & $\begin{array}{c}0.00[0.00 ; 0.01] \\
0.0 \%\end{array}$ & $0.32[0.06 ; 1.79]$ & $0.18[0.03 ; 1.01]$ & $0.65[0.12 ; 3.61]$ & $\begin{array}{c}0.17[0.00 ; 2.18] \\
62.6 \%\end{array}$ & 0.00 [0.00; 5.20] & $0.00[0.00 ; 5.35]$ \\
\hline Colitis & $\begin{array}{c}5.41[4.09 ; 6.89] \\
44.9 \%\end{array}$ & $\begin{array}{c}0.39[0.02 ; 1.04] \\
0.0 \%\end{array}$ & $\begin{array}{c}1.10[0.57 ; 1.75] \\
3.7 \%\end{array}$ & 7.19 [4.06; 12.41] & $\begin{array}{c}9.42[6.51 ; 12.77] \\
33 \%\end{array}$ & $\begin{array}{c}6.87[0.00 ; 37.07] \\
92.5 \%\end{array}$ & $\begin{array}{c}14.71[8.19 ; \\
25.00]\end{array}$ \\
\hline Diarrhea & $\begin{array}{c}5.91[4.35 ; 7.67], \\
57.6 \%\end{array}$ & $\begin{array}{c}0.92[0.19 ; 2.03] \\
34.9 \%\end{array}$ & $\begin{array}{c}0.70[0.26 ; 1.28] \\
0.0 \%\end{array}$ & 0.65 [0.12; 3.61] & $\begin{array}{c}7.20[4.31 ; 10.70] \\
47.3 \%\end{array}$ & $\begin{array}{c}2.76[0.00 ; 13.30] \\
71.2 \%\end{array}$ & $\begin{array}{c}11.76[6.08 ; \\
21.53]\end{array}$ \\
\hline Enterocolitis & $\begin{array}{c}1.25[0.00 ; 4.02] \\
84.2 \%\end{array}$ & NR & $\begin{array}{c}0.00[0.00 ; 0.21] \\
0.0 \%\end{array}$ & NR & $\begin{array}{c}0.44[0.00 ; 1.39] \\
0.0 \%\end{array}$ & $1.43[0.25 ; 7.66]$ & $0.00[0.00 ; 5.35]$ \\
\hline $\begin{array}{l}\text { Frequent bowel } \\
\text { movements }\end{array}$ & 0.00 [0.00; 1.48] & 0.00 [0.00; 1.83] & 0.00 [0.00; 0.69] & 0.00 [0.00; 2.45] & NR & NR & NR \\
\hline $\begin{array}{l}\text { Gastrointestinal } \\
\text { perforation }\end{array}$ & $\begin{array}{c}0.28[0.03 ; 0.69] \\
0.0 \%\end{array}$ & $\begin{array}{c}0.10 \text { [0.00; 0.72] } \\
16.3 \%\end{array}$ & $\begin{array}{c}0.15[0.00 ; 1.11] \\
70.1 \%\end{array}$ & NR & $\begin{array}{c}0.63[0.02 ; 1.79] \\
0.0 \%\end{array}$ & NR & NR \\
\hline
\end{tabular}


TABLE 3 | Continued

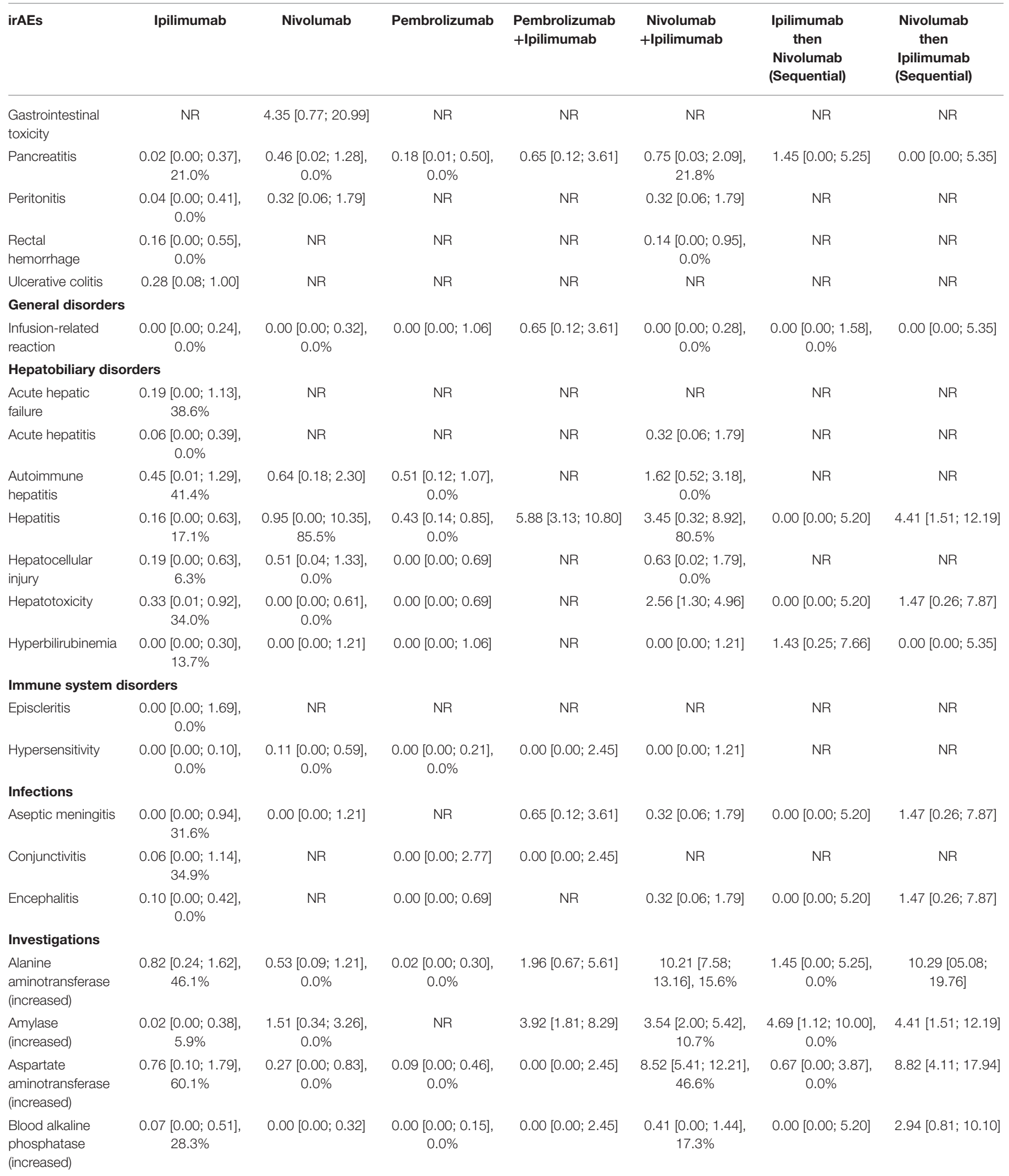

(Continued) 
TABLE 3 | Continued

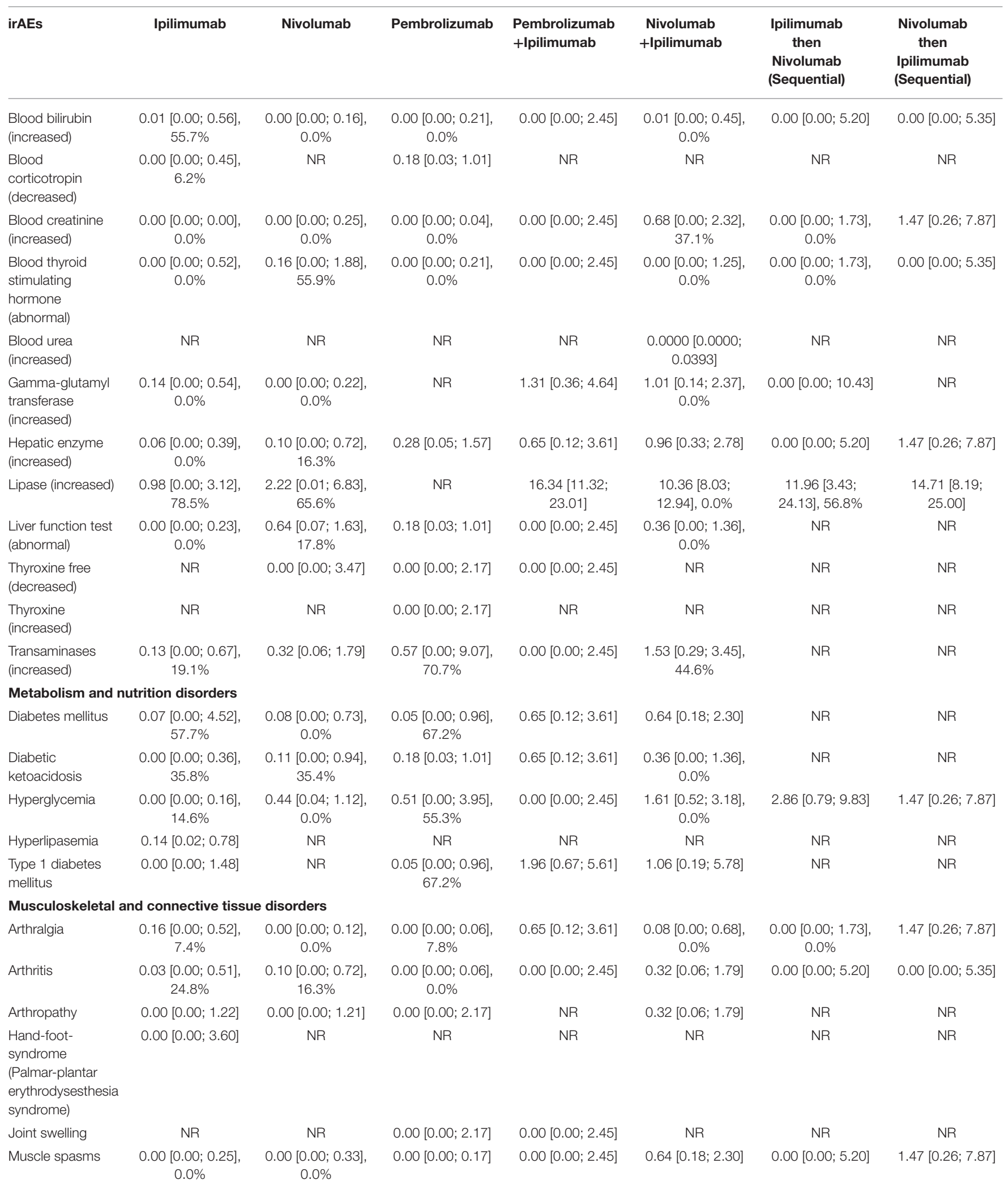


TABLE 3 | Continued

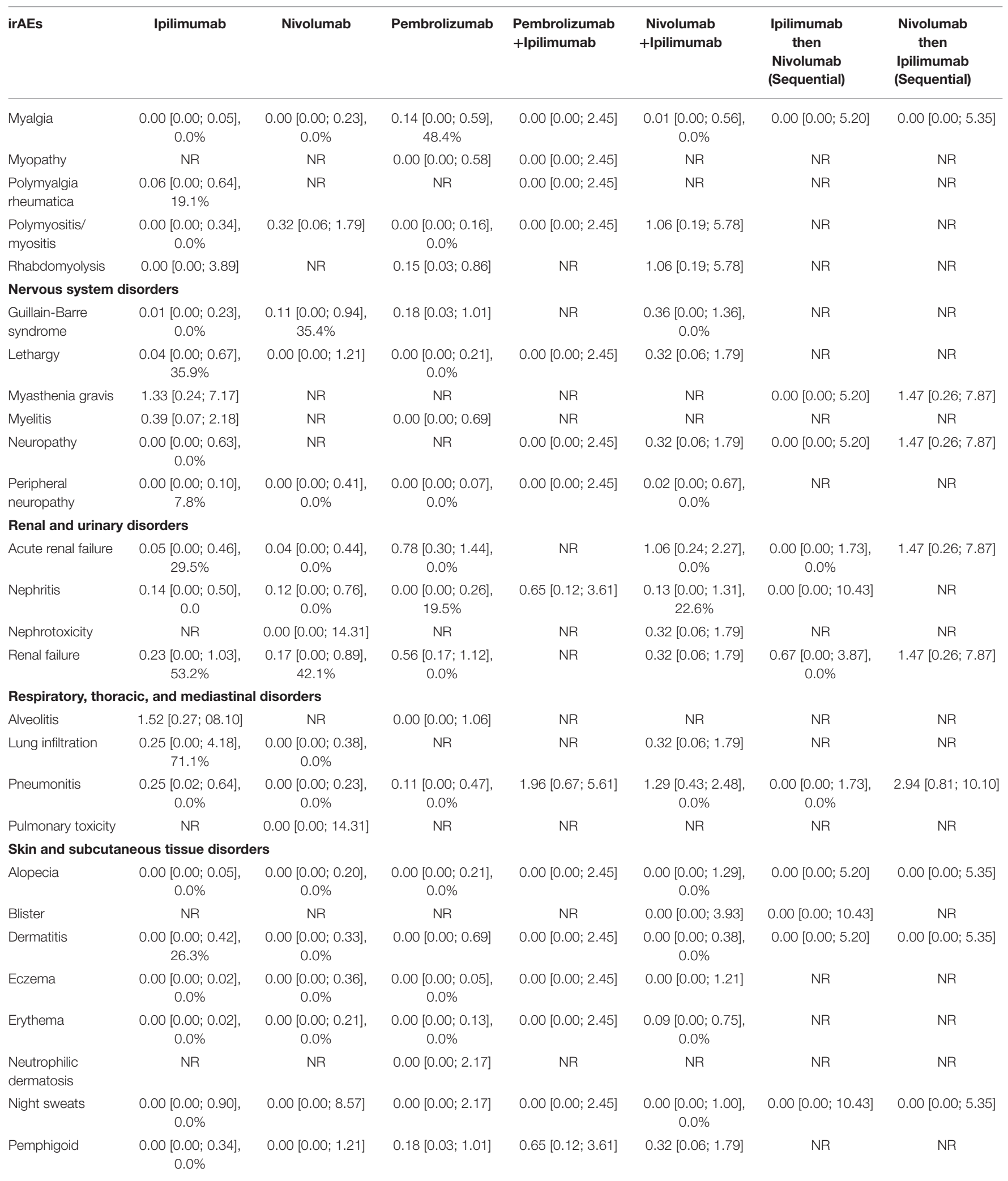


TABLE 3 | Continued

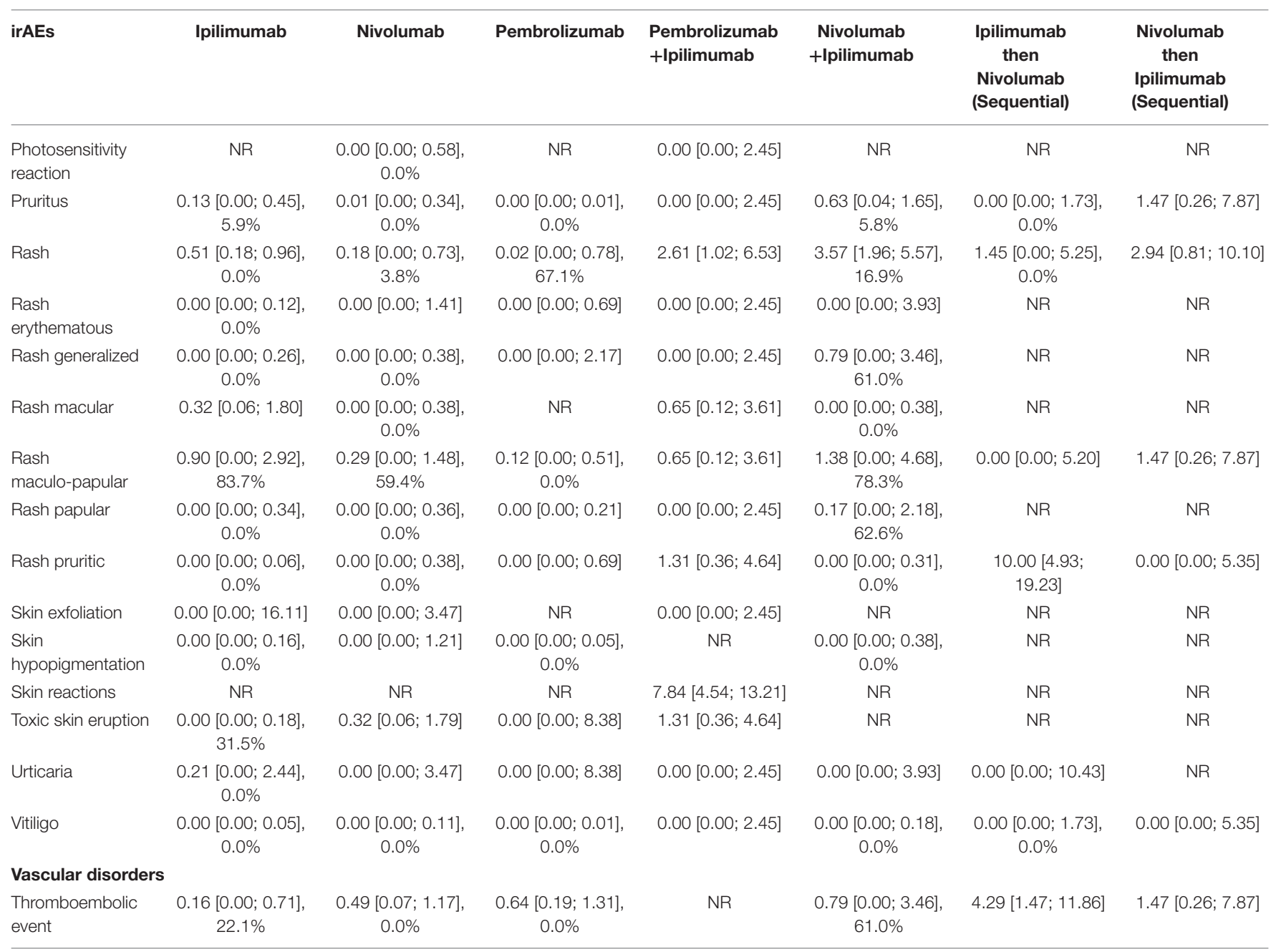

NR, not reported.

(rash and pruritus), and endocrine system (hypophysitis and hypopituitarism). In contrast, the most frequent potential irAEs of anti-PD-1 therapies involved the endocrine system (hypothyroidism, hyperthyroidism, thyroiditis, and adrenal insufficiency), gastrointestinal tract (pancreatitis and peritonitis), hepatobiliary system (hepatitis, and hepatocellular injury), endocrine system (diabetes mellitus, diabetic ketoacidosis, hyperglycemia, and type 1 diabetes mellitus), musculoskeletal system (arthritis, myalgia, and myositis), nervous system (Guillain-Barre syndrome), renal system (acute renal failure, and renal failure), and respiratory system (pneumonitis).

Our finding that the combination of anti-CTLA-4 and antiPD-1 therapies resulted in a frequent incidence of potential irAEs is consistent with the findings of three published reviews (63-65). The point estimates of the incidence of the potential irAEs in our study were slightly different from those obtained in previous meta-analyses because of different inclusion criteria; specifically, including phase1-3 ICI trials in advanced melanoma. Therefore, our analyses included a larger number of ICI studies and patients, and thus had broader evidence base and more statistical power. Furthermore, our-analysis took a step further by comprehensively profiling potential irAEs while previously reported meta-analyses were restricted to certain irAEs.

Furthermore, our findings of the most frequent incidence of potential irAEs after anti-CTLA-4 or anti-PD-1 monotherapiesespecially the higher frequency of diarrhea, colitis, rash, pruritus, hypophysitis, hypothyroidism, hyperthyroidism, and pneumonitis-are similar to the findings of previous reviews and meta-analyses $(11,63,64,66-68)$. Previous studies showed that the irAE profile might vary between nivolumab and pembrolizumab. Hypothyroidism, colitis, and pneumonitis were more common after pembrolizumab $(64,69-71)$. We also found a similar pattern among pembrolizumab users.

In our analysis, we observed a trend of elevated incidence of diabetes among nivolumab users versus pembrolizumab users. After applying a pre-specified restriction of our analysis to the FDA approved doses, the incidence of diabetes was higher in pembrolizumab users. A prior meta-analysis of endocrine adverse events of ICIs across different types of cancers showed an elevated incidence of diabetes among nivolumab users versus among pembrolizumab users (70). Although nivolumab and pembrolizumab share a similar mechanism of action, the 
TABLE 4 | Incidence of All-grades Potential Immune-Related Adverse Events (irAEs) in Advanced Melanoma presented as $\%$ with $95 \%$ confidence interval, and $l^{2}$ for the $\%$ of heterogeneity for the FDA approved doses ( $I^{2}$ not reported if the outcome was obtained from one study).

\begin{tabular}{|c|c|c|c|c|}
\hline irAEs & Ipilimumab & Nivolumab & Pembrolizumab & $\begin{array}{c}\text { Nivolumab } \\
\text { +Ipilimumab }\end{array}$ \\
\hline Number of studies & 10 & 7 & 4 & 5 \\
\hline Overall incidence & $\begin{array}{l}53.96 \text { [42.21; } \\
64.97], 94.0 \%\end{array}$ & $\begin{array}{l}44.00 \text { [31.00; } \\
57.00], 93.0 \%\end{array}$ & $\begin{array}{l}20.28 \text { [14.23; } \\
27.06], 41.0 \%\end{array}$ & $\begin{array}{l}80.00 \text { [61.00; } \\
94.00], 95.0 \%\end{array}$ \\
\hline \multicolumn{5}{|l|}{ Blood and lymphatic system disorders } \\
\hline Lymphopenia & $\begin{array}{c}2.38[0.42 ; 5.49] \\
0.0 \%\end{array}$ & $\begin{array}{c}6.76[0.00 ; 28.48] \\
81.0 \%\end{array}$ & $4.76[1.32 ; 15.79]$ & NR \\
\hline \multicolumn{5}{|l|}{ Cardiac disorders } \\
\hline Pericarditis & $0.00[0.00 ; 1.05]$ & $0.37[0.07 ; 2.08]$ & NR & $N R$ \\
\hline Ventricular arrhythmia & $0.00[0.00 ; 7.71]$ & $\begin{array}{c}0.37 \text { [0.0007; } \\
2.08]\end{array}$ & NR & $1.06[0.19 ; 5.78]$ \\
\hline \multicolumn{5}{|l|}{ Endocrine disorders } \\
\hline Acute adrenocortical insufficiency & $\begin{array}{c}0.10[0.00 ; 0.58] \\
0.0 \%\end{array}$ & $0.00[0.00 ; 1.21]$ & $0.00[0.00 ; 2.11]$ & $0.32[0.06 ; 1.79]$ \\
\hline Adrenal insufficiency & $\begin{array}{c}0.67[0.20 ; 1.32] \\
0.0 \%\end{array}$ & $\begin{array}{c}1.68[0.16 ; 4.34] \\
68.1 \%\end{array}$ & $\begin{array}{c}0.68[0.00 ; 2.24] \\
0.0 \%\end{array}$ & $\begin{array}{c}3.79[2.14 ; 5.79] \\
0.0 \%\end{array}$ \\
\hline Adrenocorticotropic hormone deficiency & $0.28[0.05 ; 1.55]$ & NR & NR & NR \\
\hline Autoimmune thyroiditis & $\begin{array}{c}0.00[0.00 ; 0.18] \\
0.0 \%\end{array}$ & $0.32[0.06 ; 1.79]$ & $N R$ & $\begin{array}{c}0.96[0.01 ; 2.90] \\
35.8 \%\end{array}$ \\
\hline Hyperthyroidism & $\begin{array}{c}0.88 \text { [0.03; 2.54] } \\
75.5 \%\end{array}$ & $\begin{array}{c}3.17[1.98 ; 4.59] \\
7.4 \%\end{array}$ & $\begin{array}{c}3.83[1.50 ; 6.97] \\
0.0 \%\end{array}$ & $\begin{array}{c}10.59 \text { [6.18; } \\
15.87], 40.9 \%\end{array}$ \\
\hline Hypophysitis & $\begin{array}{c}2.58[1.73 ; 3.57] \\
0.0 \%\end{array}$ & $\begin{array}{c}0.31[0.00 ; 1.14] \\
0.0 \%\end{array}$ & $\begin{array}{c}1.64[0.00 ; 8.01] \\
68.5 \%\end{array}$ & $\begin{array}{c}10.86 \text { [6.64; } \\
15.87], 62.8 \%\end{array}$ \\
\hline Hypopituitarism & $\begin{array}{c}0.96[0.31 ; 1.88] \\
23.4 \%\end{array}$ & $\begin{array}{c}0.16[0.00 ; 0.88] \\
4.2 \%\end{array}$ & $0.00[0.00 ; 2.11]$ & $\begin{array}{c}1.25[0.18 ; 2.95] \\
0.0 \%\end{array}$ \\
\hline Hypothyroidism & $\begin{array}{c}2.05[0.53 ; 4.28] \\
74.7 \%\end{array}$ & $\begin{array}{c}7.09[4.16 ; 10.64] \\
59.4 \%\end{array}$ & $\begin{array}{c}8.15[4.81 ; 12.17] \\
0.0 \%\end{array}$ & $\begin{array}{l}16.37 \text { [13.07; } \\
19.95], 6.4 \%\end{array}$ \\
\hline Lymphocytic hypophysitis & $\begin{array}{c}0.04[0.00 ; 0.44] \\
0.0 \%\end{array}$ & $0.00[0.00 ; 01.21]$ & NR & $0.64[0.18 ; 2.30]$ \\
\hline Thyroiditis & $\begin{array}{c}0.42[0.06 ; 1.01] \\
0.0 \%\end{array}$ & $\begin{array}{c}1.63[0.00 ; 4.96] \\
42.9 \%\end{array}$ & $N R$ & $\begin{array}{c}5.39[0.04 ; 15.93] \\
73.6 \%\end{array}$ \\
\hline Thyrotoxic crisis & $0.28[0.05 ; 1.55]$ & NR & NR & NR \\
\hline \multicolumn{5}{|l|}{ Eye disorders } \\
\hline Uveitis & $\begin{array}{c}0.14[0.00 ; 0.97] \\
52.8 \%\end{array}$ & $\begin{array}{c}0.24[0.00 ; 1.33] \\
0.0 \%\end{array}$ & $\begin{array}{c}0.04[0.00 ; 2.26] \\
0.0 \%\end{array}$ & $\begin{array}{c}2.69[0.01 ; 8.19] \\
72.1 \%\end{array}$ \\
\hline \multicolumn{5}{|l|}{ Gastrointestinal disorders } \\
\hline Autoimmune colitis & $\begin{array}{c}1.38[0.31 ; 2.99] \\
44.6 \%\end{array}$ & $0.64[0.18 ; 2.30]$ & $0.56[0.10 ; 3.11]$ & $\begin{array}{c}1.41[0.00 ; 4.98], \\
68.0 \%\end{array}$ \\
\hline Autoimmune pancreatitis & $\begin{array}{c}0.00[0.00 ; 0.22] \\
0.0 \%\end{array}$ & $0.32[0.06 ; 1.79]$ & NR & $\begin{array}{c}0.17[0.00 ; 2.18] \\
62.6 \%\end{array}$ \\
\hline Colitis & $\begin{array}{c}6.87[5.31 ; 8.58] \\
20.2 \%\end{array}$ & $\begin{array}{c}1.88[0.28 ; 4.41] \\
69.4 \%\end{array}$ & $\begin{array}{c}1.64[0.00 ; 8.01] \\
68.5 \%\end{array}$ & $\begin{array}{c}11.80 \text { [6.57; } \\
18.16], 66.3 \%\end{array}$ \\
\hline Diarrhea & $\begin{array}{l}27.74 \text { [24.28; } \\
31.33], 46.0 \%\end{array}$ & $\begin{array}{c}17.68 \text { [12.07; } \\
24.05], 76.4 \%\end{array}$ & $\begin{array}{c}11.14 \text { [6.56; } \\
16.63], 32.9 \%\end{array}$ & $\begin{array}{l}35.69 \text { [26.65; } \\
45.26], 72.7 \%\end{array}$ \\
\hline Enterocolitis & $\begin{array}{c}0.03[0.00 ; 0.51] \\
0.0 \%\end{array}$ & NR & $0.00[0.00 ; 2.11]$ & $\begin{array}{c}0.36[0.00 ; 1.36] \\
0.0 \%\end{array}$ \\
\hline Frequent bowel movements & $0.39[0.07 ; 2.18]$ & $0.97[0.27 ; 3.47]$ & NR & NR \\
\hline Gastrointestinal perforation & $\begin{array}{c}0.32 \text { [0.02; } 0.83] \\
0.0 \%\end{array}$ & $\begin{array}{c}0.10[0.00 ; 0.72] \\
16.3 \%\end{array}$ & $1.12[0.31 ; 4.00]$ & $\begin{array}{c}0.63[0.02 ; 1.79] \\
0.0 \%\end{array}$ \\
\hline Gastrointestinal toxicity & NR & $8.70[2.42 ; 26.80]$ & NR & NR \\
\hline Pancreatitis & $\begin{array}{c}0.01[0.00 ; 0.35] \\
6.0 \%\end{array}$ & $\begin{array}{c}0.62[0.07 ; 1.50] \\
0.0 \%\end{array}$ & $\begin{array}{c}0.26[0.00 ; 1.50] \\
0.0 \%\end{array}$ & $\begin{array}{c}0.75[0.05 ; 1.95] \\
0.0 \%\end{array}$ \\
\hline Peritonitis & $\begin{array}{c}0.13[0.00 ; 0.80] \\
0.0 \%\end{array}$ & $0.32[0.06 ; 1.79]$ & $N R$ & $0.32[0.06 ; 1.79]$ \\
\hline
\end{tabular}


TABLE 4 | Continued

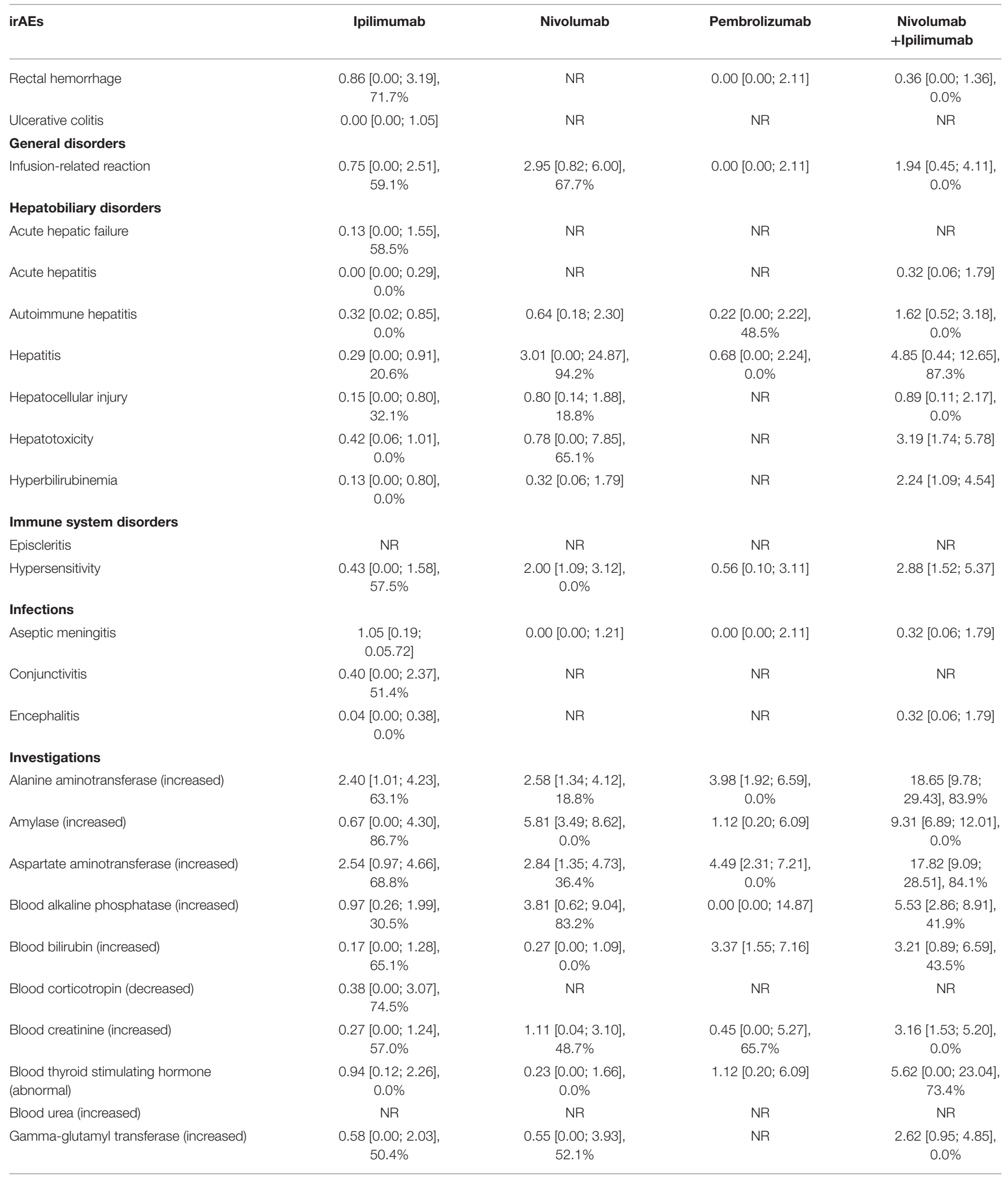


TABLE 4 | Continued

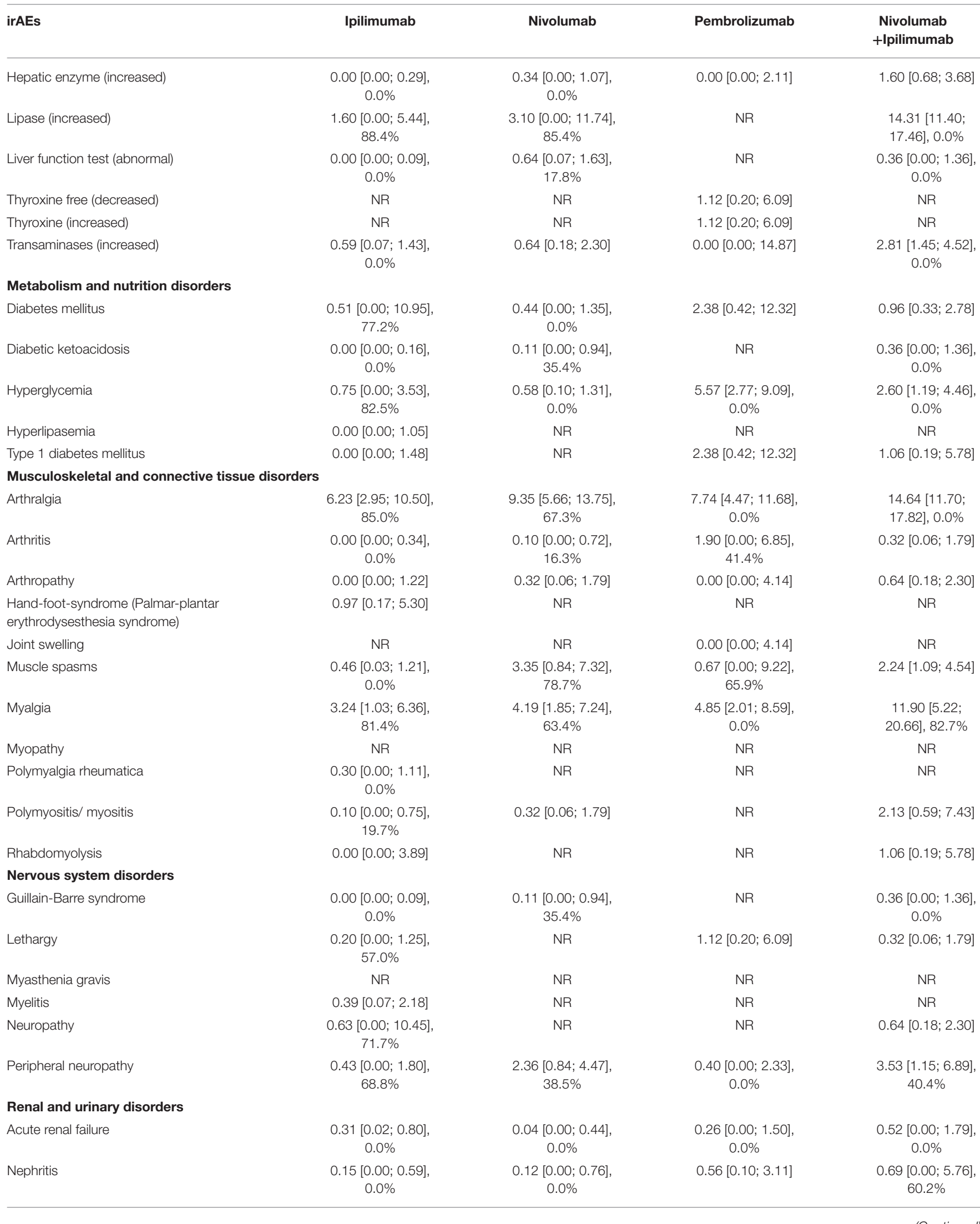

(Continued) 
TABLE 4 | Continued

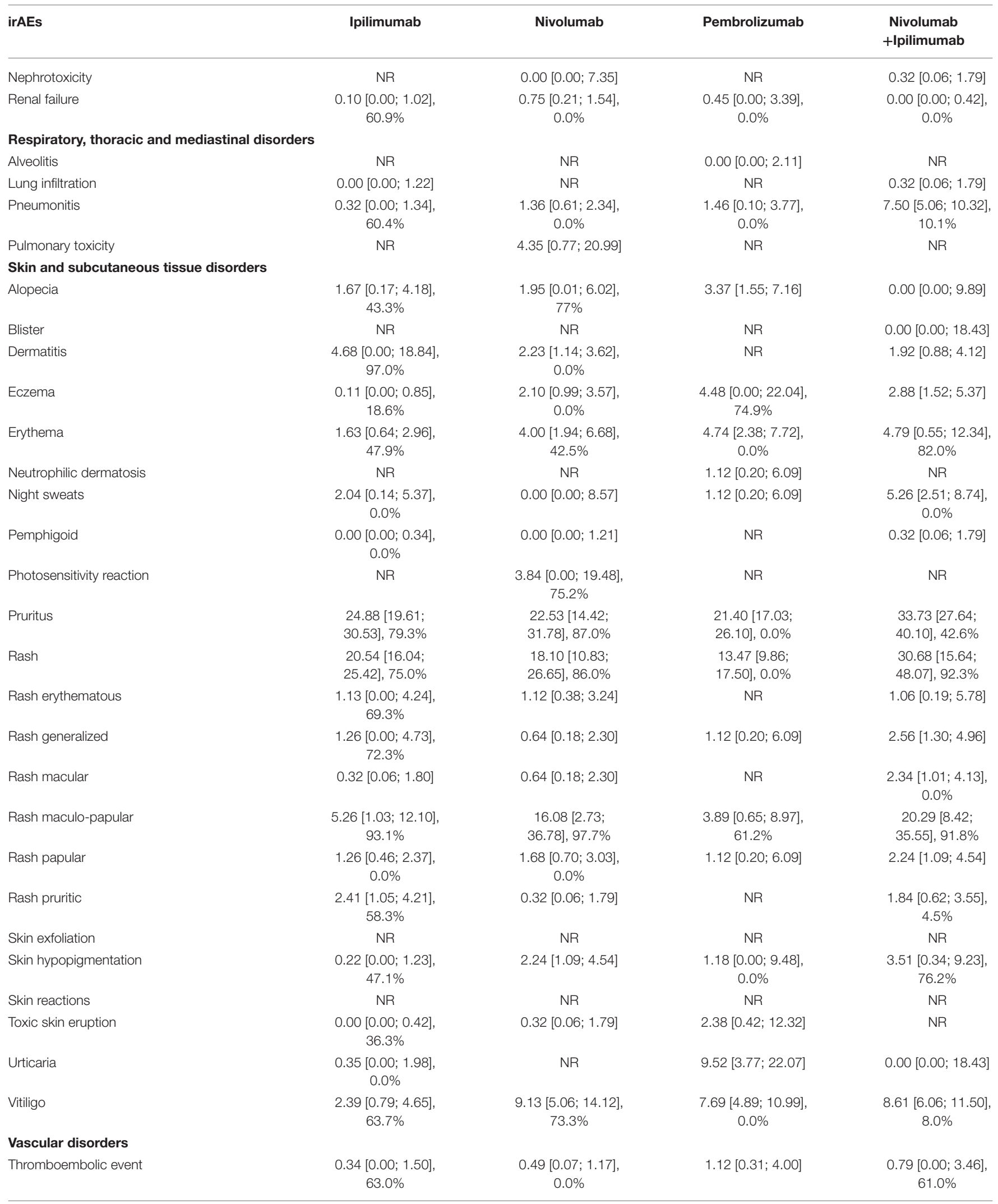

NR, not reported. 
TABLE 5 | Incidence of grade $\geq 3$ Potential Immune-Related Adverse Events (irAEs) in Advanced Melanoma presented as \% with $95 \%$ confidence interval, and ${ }^{2}$ for the $\%$ of heterogeneity for the FDA approved doses ( $l^{2}$ not reported if the outcome was obtained from one study).

\begin{tabular}{|c|c|c|c|c|}
\hline irAEs & Ipilimumab & Nivolumab & Pembrolizumab & $\begin{array}{c}\text { Nivolumab } \\
\text { +Ipilimumab }\end{array}$ \\
\hline Number of studies & 10 & 7 & 4 & 5 \\
\hline Overall incidence & $\begin{array}{l}11.95[10.17 \\
13.85], 7.0 \%\end{array}$ & $\begin{array}{c}4.00[1.00 ; 7.00] \\
70.0 \%\end{array}$ & $\begin{array}{c}1.65[0.33 ; 3.59] \\
0.0 \%\end{array}$ & $\begin{array}{l}32.00 \text { [16.00; } \\
49.00], 93.0 \%\end{array}$ \\
\hline \multicolumn{5}{|l|}{ Blood and lymphatic system disorders } \\
\hline Lymphopenia & $0.00[0.00 ; 1.80]$ & $\begin{array}{c}2.20[0.00 ; 24.35] \\
87 \%\end{array}$ & $\begin{array}{c}0.00[0.0000 \\
0.0838]\end{array}$ & NR \\
\hline \multicolumn{5}{|l|}{ Cardiac disorders } \\
\hline Pericarditis & $0.00[0.00 ; 1.05]$ & $0.37[0.07 ; 2.08]$ & NR & $N R$ \\
\hline Ventricular arrhythmia & $0.00[0.00 ; 7.71]$ & $0.37[0.07 ; 2.08]$ & NR & $0.00[0.00 ; 3.93]$ \\
\hline \multicolumn{5}{|l|}{ Endocrine disorders } \\
\hline Acute adrenocortical insufficiency & $\begin{array}{c}0.10[0.00 ; 0.58] \\
0.0 \%\end{array}$ & $0.00[0.00 ; 1.21]$ & $0.00[0.00 ; 2.11]$ & $0.32[0.06 ; 1.79]$ \\
\hline Adrenal insufficiency & $\begin{array}{c}0.00[0.00 ; 0.16] \\
0.0 \%\end{array}$ & $\begin{array}{c}0.77[0.07 ; 1.97] \\
25.2 \%\end{array}$ & $\begin{array}{c}0.68[0.00 ; 2.24] \\
0.0 \%\end{array}$ & $\begin{array}{c}1.13[0.22 ; 2.48] \\
0.0 \%\end{array}$ \\
\hline Adrenocorticotropic hormone deficiency & $0.00[0.00 ; 1.05]$ & NR & NR & NR \\
\hline Autoimmune thyroiditis & $\begin{array}{c}0.00[0.00 ; 0.18] \\
0.0 \%\end{array}$ & $0.00[0.00 ; 1.21]$ & NR & $\begin{array}{c}0.36[0.00 ; 1.36] \\
0.0 \%\end{array}$ \\
\hline Hyperthyroidism & $\begin{array}{c}0.04[0.00 ; 0.38] \\
0.0 \%\end{array}$ & $\begin{array}{c}0.00[0.00 ; 0.22] \\
0.0 \%\end{array}$ & $\begin{array}{c}0.00[0.00 ; 0.61] \\
0.0 \%\end{array}$ & $\begin{array}{c}0.52[0.00 ; 1.73] \\
0.0 \%\end{array}$ \\
\hline Hypophysitis & $\begin{array}{c}1.21[0.61 ; 1.97] \\
0.0 \%\end{array}$ & $\begin{array}{c}0.15[0.00 ; 0.87] \\
0.0 \%\end{array}$ & $\begin{array}{c}0.68[0.00 ; 3.10] \\
19.1 \%\end{array}$ & $\begin{array}{c}2.48[0.72 ; 4.97] \\
34.0 \%\end{array}$ \\
\hline Hypopituitarism & $\begin{array}{c}0.63[0.07 ; 1.55] \\
36.0 \%\end{array}$ & $\begin{array}{c}0.08[0.00 ; 0.71] \\
0.0 \%\end{array}$ & $0.00[0.00 ; 2.11]$ & $\begin{array}{c}0.43[0.00 ; 1.71] \\
0.0 \%\end{array}$ \\
\hline Hypothyroidism & $\begin{array}{c}0.00[0.00 ; 0.00] \\
0.0 \%\end{array}$ & $\begin{array}{c}0.00[0.00 ; 0.00] \\
0.0 \%\end{array}$ & $\begin{array}{c}0.00[0.00 ; 0.36] \\
0.0 \%\end{array}$ & $\begin{array}{c}0.00[0.00 ; 0.54] \\
0.0 \%\end{array}$ \\
\hline Lymphocytic hypophysitis & $\begin{array}{c}0.00[0.00 ; 0.20] \\
0.0 \%\end{array}$ & $0.00[0.00 ; 01.21]$ & NR & $0.32[0.06 ; 1.79]$ \\
\hline Thyroiditis & $\begin{array}{c}0.00[0.00 ; 0.22] \\
0.0 \%\end{array}$ & $\begin{array}{c}0.00[0.00 ; 0.15] \\
0 \%\end{array}$ & $N R$ & $\begin{array}{c}0.00[0.00 ; 0.41] \\
0.0 \%\end{array}$ \\
\hline Thyrotoxic crisis & $0.28[0.05 ; 1.55]$ & NR & NR & NR \\
\hline \multicolumn{5}{|l|}{ Eye disorders } \\
\hline Uveitis & $\begin{array}{c}0.02 \text { [0.00; } 0.70] \\
49.4 \%\end{array}$ & $\begin{array}{c}0.00[0.00 ; 0.22] \\
0.0 \%\end{array}$ & $\begin{array}{c}0.00[0.00 ; 0.36] \\
0.0 \%\end{array}$ & $\begin{array}{c}0.42[0.00 ; 3.95] \\
70.1 \%\end{array}$ \\
\hline \multicolumn{5}{|l|}{ Gastrointestinal disorders } \\
\hline Autoimmune colitis & $\begin{array}{c}0.81[0.17 ; 1.75] \\
10.2 \%\end{array}$ & $0.32[0.06 ; 1.79]$ & $0.56[0.10 ; 3.11]$ & $\begin{array}{c}1.19[0.00 ; 5.54] \\
77.8 \%\end{array}$ \\
\hline Autoimmune pancreatitis & $\begin{array}{c}0.00[0.00 ; 0.22] \\
0.0 \%\end{array}$ & $0.32[0.06 ; 1.79]$ & NR & $\begin{array}{c}0.17[0.00 ; 2.18] \\
62.6 \%\end{array}$ \\
\hline Colitis & $\begin{array}{c}4.69 \text { [3.06; } 6.59], \\
43.8 \%\end{array}$ & $\begin{array}{c}0.33[0.00 ; 1.00] \\
0.0 \%\end{array}$ & $\begin{array}{c}0.34[0.00 ; 4.92] \\
66.7 \%\end{array}$ & $\begin{array}{c}9.02[5.12 ; 13.76] \\
51.8 \%\end{array}$ \\
\hline Diarrhea & $\begin{array}{c}4.50[3.03 ; 6.21] \\
37.5 \%\end{array}$ & $\begin{array}{c}0.52[0.00 ; 1.60] \\
30.6 \%\end{array}$ & $\begin{array}{c}0.00[0.00 ; 0.30] \\
0.0 \%\end{array}$ & $\begin{array}{c}5.52[2.24 ; 9.89], \\
58.4 \%\end{array}$ \\
\hline Enterocolitis & $\begin{array}{c}0.03 \text { [0.00; } 0.51] \\
0.0 \%\end{array}$ & NR & $0.00[0.00 ; 2.11]$ & $\begin{array}{c}0.36[0.00 ; 1.36] \\
0.0 \%\end{array}$ \\
\hline Frequent bowel movements & $0.00[0.00 ; 1.48]$ & $0.00[0.00 ; 1.83]$ & NR & NR \\
\hline Gastrointestinal perforation & $\begin{array}{c}0.32[0.02 ; 0.83] \\
0.0 \%\end{array}$ & $\begin{array}{c}0.10[0.00 ; 0.72] \\
16.3 \%\end{array}$ & $1.12[0.31 ; 4.00]$ & $\begin{array}{c}0.63[0.02 ; 1.79] \\
0.0 \%\end{array}$ \\
\hline Gastrointestinal toxicity & NR & $4.35[0.77 ; 20.99]$ & NR & NR \\
\hline Pancreatitis & $\begin{array}{c}0.01[0.00 ; 0.35] \\
6.0 \%\end{array}$ & $\begin{array}{c}0.46[0.02 ; 1.28] \\
0.0 \%\end{array}$ & $\begin{array}{c}0.00[0.00 ; 0.67] \\
0.0 \%\end{array}$ & $\begin{array}{c}0.20[0.00 ; 1.08] \\
0.0 \%\end{array}$ \\
\hline
\end{tabular}


TABLE 5 | Continued

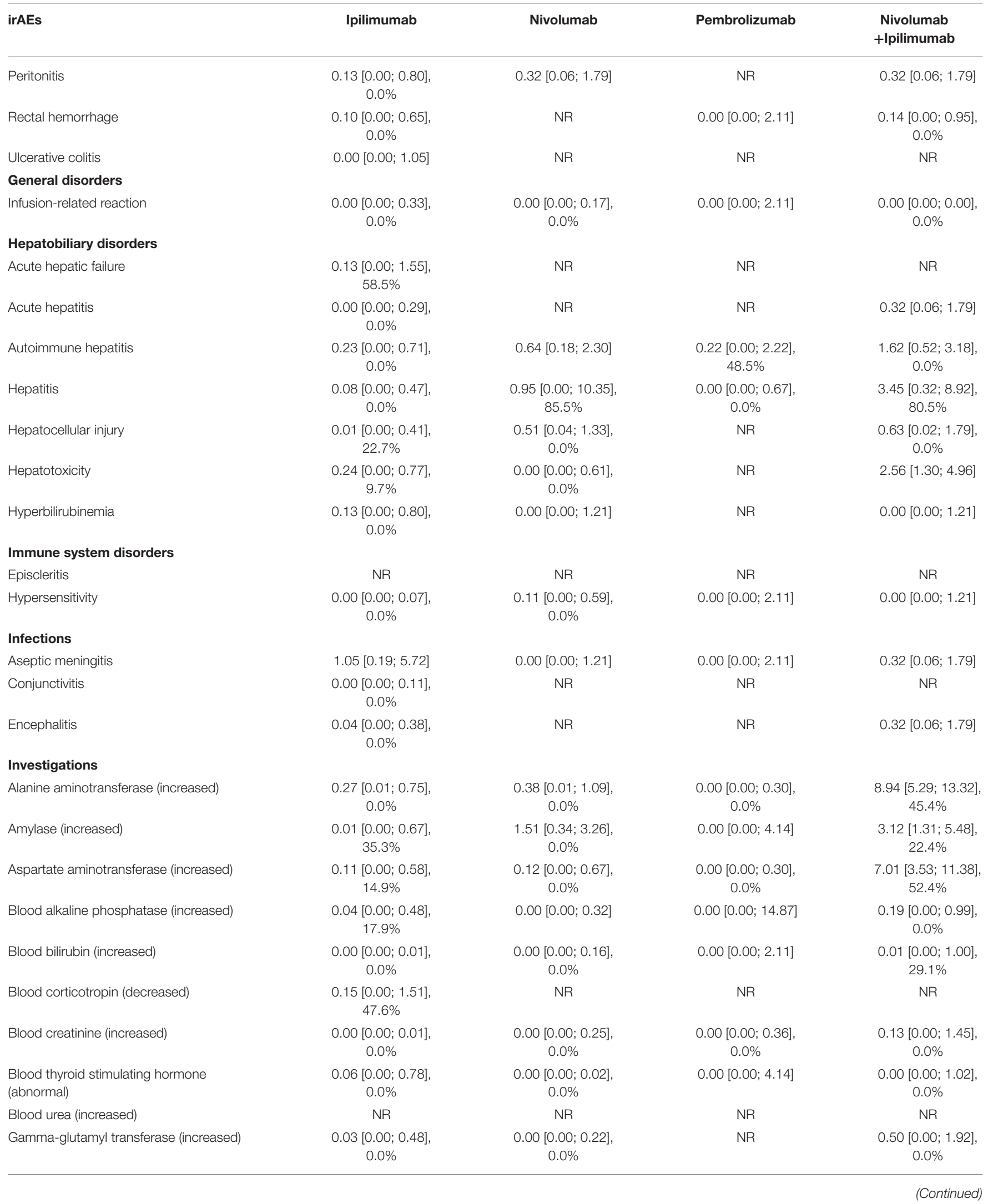


TABLE 5 | Continued

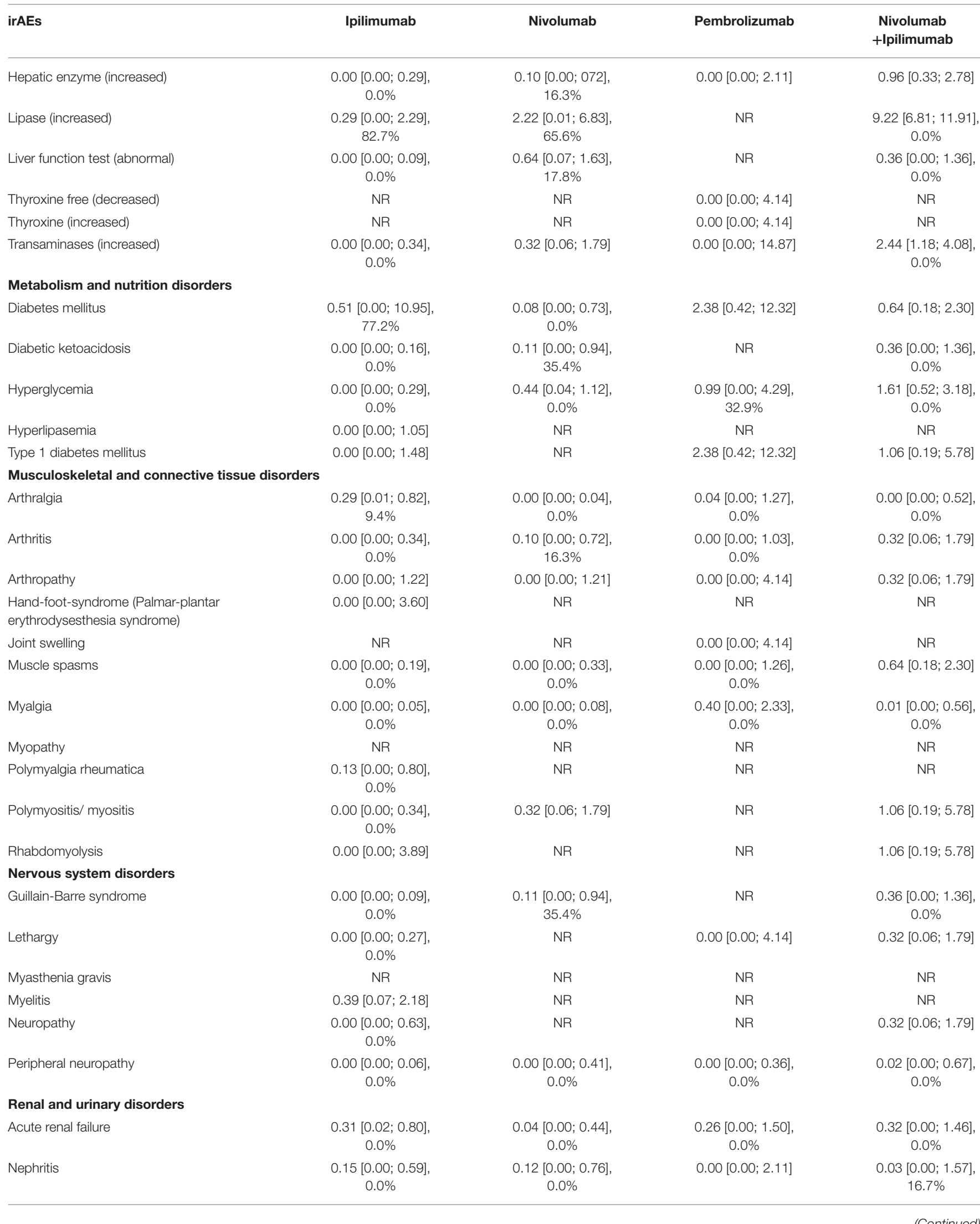

(Continued) 
TABLE 5 | Continued

\begin{tabular}{|c|c|c|c|c|}
\hline irAEs & Ipilimumab & Nivolumab & Pembrolizumab & $\begin{array}{c}\text { Nivolumab } \\
\text { +Ipilimumab }\end{array}$ \\
\hline Nephrotoxicity & NR & 0.00 [0.00; 7.35] & NR & $0.32[0.06 ; 1.79]$ \\
\hline \multicolumn{5}{|c|}{ Respiratory, thoracic, and mediastinal disorders } \\
\hline Alveolitis & NR & NR & $\begin{array}{c}0.00 \text { [0.00; } \\
0.0211]\end{array}$ & NR \\
\hline Pneumonitis & $\begin{array}{c}0.07 \text { [0.00; } 0.44] \\
0.0 \%\end{array}$ & $\begin{array}{c}0.00[0.00 ; 0.25] \\
0.0 \%\end{array}$ & $\begin{array}{c}0.27[0.00 ; 1.85] \\
0.0 \%\end{array}$ & $\begin{array}{c}0.81[0.08 ; 2.01] \\
0.0 \%\end{array}$ \\
\hline Pulmonary toxicity & NR & $0.00[0.00 ; 14.31]$ & NR & NR \\
\hline \multicolumn{5}{|c|}{ Skin and subcutaneous tissue disorders } \\
\hline Alopecia & $\begin{array}{c}0.00 \text { [0.00; } 0.09] \\
0.0 \%\end{array}$ & $\begin{array}{c}0.00[0.00 ; 0.20] \\
0.0 \%\end{array}$ & $0.00[0.00 ; 2.11]$ & 0.00 [0.00; 9.89] \\
\hline Eczema & $\begin{array}{c}0.00[0.00 ; 0.02] \\
0.0 \%\end{array}$ & $\begin{array}{c}0.00[0.00 ; 0.36] \\
0.0 \%\end{array}$ & $\begin{array}{c}0.00[0.00 ; 2.84] \\
0.0 \%\end{array}$ & $0.00[0.00 ; 121]$ \\
\hline Erythema & $\begin{array}{c}0.00[0.00 ; 0.17] \\
0.0 \%\end{array}$ & $\begin{array}{c}0.00[0.00 ; 0.23] \\
0.0 \%\end{array}$ & $\begin{array}{c}0.00[0.00 ; 0.32] \\
0.0 \%\end{array}$ & $\begin{array}{c}0.14[0.00 ; 0.95] \\
0.0 \%\end{array}$ \\
\hline Neutrophilic dermatosis & NR & NR & $0.00[0.00 ; 4.14]$ & NR \\
\hline Night sweats & $\begin{array}{c}0.00[0.00 ; 1.27] \\
0.0 \%\end{array}$ & $0.00[0.00 ; 8.57]$ & $0.00[0.00 ; 4.14]$ & $\begin{array}{l}0.00[0.00 ; \\
0.50], 0.0 \%\end{array}$ \\
\hline Pemphigoid & $\begin{array}{c}0.00 \text { [0.00; } 0.34] \\
0.0 \%\end{array}$ & $0.00[0.00 ; 1.21]$ & NR & $0.32[0.06 ; 1.79]$ \\
\hline Photosensitivity reaction & NR & $\begin{array}{c}0.00[0.00 ; 0.12] \\
0.0 \%\end{array}$ & NR & NR \\
\hline Pruritus & $\begin{array}{c}0.05 \text { [0.00; } 0.39] \\
0.0 \%\end{array}$ & $\begin{array}{c}0.00[0.00 ; 0.22] \\
0.0 \%\end{array}$ & $\begin{array}{c}0.00 \text { [0.00; } 0.88] \\
21.9 \%\end{array}$ & $\begin{array}{c}0.57[0.01 ; 1.65] \\
0.0 \%\end{array}$ \\
\hline Rash macular & $0.32[0.06 ; 1.80]$ & $0.00[0.00 ; 1.21]$ & NR & $\begin{array}{c}0.17[0.00 ; 2.18] \\
0.0 \%\end{array}$ \\
\hline Rash maculo-papular & $\begin{array}{c}0.03 \text { [0.00; } 0.36] \\
0.0 \%\end{array}$ & $\begin{array}{c}0.43[0.00 ; 2.05] \\
66.3 \%\end{array}$ & $\begin{array}{c}0.02[0.00 ; 0.88] \\
0.0 \%\end{array}$ & $\begin{array}{c}2.14[0.00 ; 6.83], \\
81.0 \%\end{array}$ \\
\hline Rash papular & $\begin{array}{c}0.00[0.00 ; 0.34] \\
0.0 \%\end{array}$ & $\begin{array}{l}0.00[0.00 ; \\
0.36], 0.0 \%\end{array}$ & $0.00[0.00 ; 4.14]$ & $0.00[0.00 ; 1.21]$ \\
\hline Rash pruritic & $\begin{array}{c}0.00 \text { [0.00; } 0.22] \\
0.0 \%\end{array}$ & $0.00[0.00 ; 1.21]$ & NR & $\begin{array}{c}0.00[0.00 ; 0.38] \\
0.0 \%\end{array}$ \\
\hline Skin exfoliation & NR & NR & NR & NR \\
\hline Skin hypopigmentation & $\begin{array}{c}0.00 \text { [0.00; } 0.34] \\
0.0 \%\end{array}$ & $0.00[0.00 ; 1.21]$ & $\begin{array}{c}0.00[0.00 ; 1.36] \\
0.0 \%\end{array}$ & $\begin{array}{c}0.00[0.00 ; 0.38] \\
0.0 \%\end{array}$ \\
\hline Skin reactions & NR & NR & $N R$ & NR \\
\hline Toxic skin eruption & $\begin{array}{c}0.00 \text { [0.00; } 0.42] \\
36.3 \%\end{array}$ & $0.32[0.06 ; 1.79]$ & $0.00[0.00 ; 8.38]$ & NR \\
\hline Urticaria & $0.00[0.00 ; 5.13]$ & NR & 0.00 [0.00; 8.38] & 0.00 [0.00; 18.43] \\
\hline Vitiligo & $\begin{array}{c}0.00[0.00 ; 0.14] \\
0.0 \%\end{array}$ & $\begin{array}{c}0.00[0.00 ; 0.03] \\
0.0 \%\end{array}$ & $\begin{array}{c}0.00[0.00 ; 0.30] \\
0.0 \%\end{array}$ & $\begin{array}{c}0.00[0.00 ; 0.04] \\
0.0 \%\end{array}$ \\
\hline \multicolumn{5}{|l|}{ Vascular disorders } \\
\hline Thromboembolic event & $\begin{array}{c}0.19[0.00 ; 0.91] \\
0.0 \%\end{array}$ & $\begin{array}{c}0.49[0.07 ; 1.17] \\
0.0 \%\end{array}$ & $0.00[0.00 ; 2.11]$ & $\begin{array}{c}0.79[0.00 ; 3.46] \\
61.0 \%\end{array}$ \\
\hline
\end{tabular}

NR, not reported. 
incidence of diabetes can be triggered by other factors such as the cancer types and the presence of genetic factors or autoimmune bodies. A recent meta-analysis by $\mathrm{Lu}$ et al. showed that the incidence of diabetes due to ICIs differs from one cancer type to another (72). Several studies have concluded that patients with an underlying genetic predisposition to diabetes or having autoimmune bodies such as antiglutamic acid decarboxylase 65 (GAD) antibody, are at risk of developing diabetes following treatment with anti-PD1 therapies (73-75). Moreover, subgroup analysis for the FDA doses reduced the number of studies as well as the sample of patients treated with ICIs, which may have affected the estimation of the risk of the incidence of diabetes (76).

Although a previous meta-analysis showed a trend of a high incidence of elevated levels of ALT and AST in melanoma patients treated with nivolumab versus pembrolizumab (69), our analyses revealed a higher incidence of these potential irAEs in patients treated with pembrolizumab. Note that our estimates of the incidence of elevated levels of ALT and AST was based on five pembrolizumab studies (1262 patients) and six nivolumab studies (996 patients), whereas the prior meta-analysis involved only two pembrolizumab studies (173 patients) and five nivolumab studies (955 patients). This differences in the number of studies and the higher aggregate of patients observed may explain the variation in the estimated incidence of these potential irAEs in our analysis compared to the previous meta-analysis (76).

Our findings are important for clinicians as they provide valuable information about the expected frequencies of potential irAEs in patients with advanced melanoma treated with antiCTLA-4 or anti-PD-1 monotherapies or combination therapies. In the current study, the overall incidence of each potential irAE was presented across different doses as well as for the FDAapproved doses of ICIs. Moreover, the findings of our study could help clinicians including non-oncologists such as primary care providers be vigilant about serious potential irAEs and to raise their awareness about the expected incidence rates of potential irAEs. Also, our findings provide a safety reference of ICIs in advanced melanoma against which new data such as real-world data can be compared.

Our study has some strengths. To the best of our knowledge, the current meta-analysis is the first that was limited to advanced melanoma, included different combination of ICIs, and comprehensively presented the frequencies of potential irAEs for FDA-approved doses of ICIs. We used data from the regulatory bodies and clinicaltrials.gov to obtain more comprehensive data about potential irAEs, especially for trials that were not published as journal articles. Moreover, we included patients treated with ICI monotherapy or a combination of ICIs (antiCTLA-4 and anti-PD-1) and excluded any combination of other active treatments to accurately estimate the incidence of potential irAEs.

This study nevertheless has some limitations. Classifying an adverse event as a potential irAE is very challenging and may vary across trials or observers, which may lead to detection bias because we included open-label trials as well as double-blind trials. These variations may lead to overestimating the incidence of potential irAEs. However, we included only patients treated with anti-CTLA-4 or anti-PD-1 monotherapies or combination therapies to avoid including AEs that could be related to other therapies or other immunotherapies. Moreover, we utilized the random-effect model for the meta-analyses as well as subgroup analyses for high heterogeneity to account for possible variation in the outcomes of the included studies $(77,78)$. In addition, the use of trial-level data rather than patient-level data may have introduced bias in estimating the incidence of potential irAEs owing to the variation in patient characteristics, which may be associated with the occurrence of potential irAEs. While we agree that identifying modifiers from patient-level data tends to be more robust than using trial-level data, we performed subgroup analyses to explore heterogeneity based on the available information on possible modifiers reported at the trial level (79).

\section{CONCLUSIONS}

Overall, we found that the incidence of potential irAEs was high after using a combination of anti-CTLA-4 and anti-PD1 therapies. Ipilimumab users tended to be at a higher risk of developing diarrhea, colitis, rash, pruritus, and hypophysitis than anti-PD-1 users. Pembrolizumab users were at a high risk of potential irAEs including thyroid disorders (hypothyroidism and hypothyroidism), endocrine disorders (hyperglycemia, and type 1 diabetes mellitus), respiratory disorders (pneumonitis), skin disorders (eczema, toxic skin eruptions, urticaria, and vitiligo), and musculoskeletal disorders (myalgia and arthralgia). Nivolumab users were likely to have a high incidence of maculopapular rash, erythema, hepatitis, infusion-related reaction, hypersensitivity, thyroiditis, elevated levels of amylase and lipase, muscle spasms, peripheral neuropathy, and renal failure. Accordingly, clinicians should be cognizant of variations in the potential irAE profiles of ICIs and weigh the benefits and risks of irAEs associated with these agents as mono or combination therapies. Nevertheless, further studies are needed to identify key predictors associated with the occurrence of irAEs as well as to compare their incidences in the real-world setting to help clinicians personalize treatment for patients and effectively manage any potential irAEs.

\section{DATA AVAILABILITY STATEMENT}

All the analyzed data in this study were included in the published article.

\section{AUTHOR CONTRIBUTIONS}

All authors: study concept and design, critical revision of the manuscript, and interpretation of data. AA: data acquisition and management and statistical analysis. AA and IA: drafting of the manuscript. IA: study supervision.

\section{SUPPLEMENTARY MATERIAL}

The Supplementary Material for this article can be found online at: https://www.frontiersin.org/articles/10.3389/fonc. 2020.00091/full\#supplementary-material 


\section{REFERENCES}

1. Siegel RL, Miller KD, Jemal A. Cancer statistics, 2019. CA Cancer J Clin. (2019) 69:7-34. doi: 10.3322/caac.21551

2. Bray F, Ferlay J, Soerjomataram I, Siegel RL, Torre LA, Jemal A. Global cancer statistics 2018: GLOBOCAN estimates of incidence and mortality worldwide for 36 cancers in 185 countries. CA Cancer J Clin. (2018) 68:394424. doi: $10.3322 /$ caac. 21492

3. Seidel JA, Otsuka A, Kabashima K. Anti-PD-1 and Anti-CTLA-4 Therapies in cancer: mechanisms of action, efficacy, and limitations. Front Oncol. (2018) 8:86. doi: $10.3389 /$ fonc. 2018.00086

4. Traynor K. Ipilimumab approved for metastatic melanoma. Am J Health Syst Pharm. (2011) 68:768. doi: 10.2146/news110025

5. Raedler LA. Keytruda (Pembrolizumab): first PD-1 inhibitor approved for previously treated unresectable or metastatic melanoma. Am Health Drug Benefits. (2015) 8:96-100.

6. Raedler LA. Opdivo (Nivolumab): second PD-1 inhibitor receives FDA approval for unresectable or metastatic melanoma. Am Health Drug Benefits. (2015) 8:180-3.

7. Carlino MS, Long GV. Ipilimumab combined with nivolumab: a standard of care for the treatment of advanced melanoma? Clin Cancer Res. (2016) 22:3992-8. doi: 10.1158/1078-0432.CCR-15-2944

8. Brahmer JR, Lacchetti C, Schneider BJ, Atkins MB, Brassil KJ, Caterino $\mathrm{JM}$, et al. Management of immune-related adverse events in patients treated with immune checkpoint inhibitor therapy: American society of clinical oncology clinical practice guideline. J Clin Oncol. (2018) 36:171468. doi: 10.1200/JCO.2017.77.6385

9. Haanen J, Carbonnel F, Robert C, Kerr KM, Peters S, Larkin J, et al. Management of toxicities from immunotherapy: ESMO Clinical Practice Guidelines for diagnosis, treatment and follow-up. Ann Oncol. (2018) 29:iv264-6. doi: 10.1093/annonc/mdy162

10. NationalComprehensiveCancerNetwork(NCCN). NCCN Clinical Practice Guidelines in Oncology (NCCN Guidelines $(\mathrm{R})$, in partnership with the American Society of Clinical Oncology (ASCO) Management of Immunotherapy-Related Toxicity (Version 1.2019). Available online at: https:// www.nccn.org/professionals/physician_gls/pdf/immunotherapy.pdf

11. Michot JM, Bigenwald C, Champiat S, Collins M, Carbonnel F, Postel-Vinay S, et al. Immune-related adverse events with immune checkpoint blockade: a comprehensive review. Eur J Cancer. (2016) 54:139-48. doi: 10.1016/j.ejca.2015.11.016

12. Duma N, Azzouqa A, Yadav S, Hoversten K, Reed C, Sitek A, et al. 1218P Immune-related adverse events: Comparison of melanoma and non-small cell lung cancer patients treated with anti-PD1 therapy. Ann Oncol. (2018) 29(Suppl_8):mdy288. 089. doi: 10.1093/annonc/mdy 288.089

13. Owen D, Burkart J, Patel S, Wei L, Tinoco G, Liebner D, et al. 1772P Immune related adverse events across cancer types: Incidence, risk factors and survival. Ann Oncol. (2018) 29(suppl_8):mdy300. 088. doi: 10.1093/annonc/mdy300.088

14. Irwin DE, Davis B, Bell JA, Galaznik A, Garcia-Ribas I. Gastrointestinal complications in patients treated with ipilimumab and nivolumab combination therapy or monotherapy. J Comp Eff Res. (2019) 8:81-90. doi: 10.2217/cer-2018-0072

15. Ma K, Lu Y, Jiang S, Tang J, Li X, Zhang Y. The relative risk and incidence of immune checkpoint inhibitors related pneumonitis in patients with advanced cancer: a meta-analysis. Front Pharmacol. (2018) 9:1430. doi: 10.3389/fphar.2018.01430

16. Khoja L, Day D, Wei-Wu Chen T, Siu LL, Hansen AR. Tumourand class-specific patterns of immune-related adverse events of immune checkpoint inhibitors: a systematic review. Ann Oncol. (2017) 28:2377-85. doi: 10.1093/annonc/mdx286

17. Moher D, Liberati A, Tetzlaff J, Altman DG, Group P. Preferred reporting items for systematic reviews and meta-analyses: the PRISMA Statement. Open Med. (2009) 3:e123-30. doi: 10.1371/journal.pmed. 1000097

18. Higgins JP, Altman DG, Gotzsche PC, Juni P, Moher D, Oxman AD, et al. The Cochrane Collaboration's tool for assessing risk of bias in randomised trials. BMJ. (2011) 343:d5928. doi: 10.1136/bmj.d5928
19. Wells G, Shea B, O'Connell J. The Newcastle-Ottawa Scale (NOS) for Assessing the Quality of Nonrandomised Studies in Meta-analyses. The Ottawa Hospital Research Institute (2014).

20. Rousseau MJ, Evans JC. Key statistical assumptions and methods in one-arm meta-analyses with binary endpoints and low event rates, including a reallife example in the area of endoscopic colonic stenting. Cogent Med. (2017) 4:1334318. doi: 10.1080/2331205X.2017.1334318

21. Agresti A, Coull BA. Approximate is better than "exact" for interval estimation of binomial proportions. Am Statist. (1998) 52:119-26. doi: 10.1080/00031305.1998.10480550

22. Weber JS, Gibney G, Sullivan RJ, Sosman JA, Slingluff CL, Jr., Lawrence $\mathrm{DP}$, et al. Sequential administration of nivolumab and ipilimumab with a planned switch in patients with advanced melanoma (CheckMate 064): an open-label, randomised, phase 2 trial. Lancet Oncol. (2016) 17:94355. doi: 10.1016/S1470-2045(16)30126-7

23. Wolchok JD, Chiarion-Sileni V, Gonzalez R, Rutkowski P, Grob JJ, Cowey CL et al. Overall Survival with combined nivolumab and ipilimumab in advanced melanoma. N Engl J Med. (2017) 377:1345-56. doi: 10.1056/NEJMoa1709684

24. Long GV, Atkinson V, Lo S, Sandhu S, Guminski AD, Brown MP, et al. Combination nivolumab and ipilimumab or nivolumab alone in melanoma brain metastases: a multicentre randomised phase 2 study. Lancet Oncol. (2018) 19:672-81. doi: 10.1016/S1470-2045(18)30139-6

25. Tawbi HA, Forsyth PA, Algazi A, Hamid O, Hodi FS, Moschos SJ, et al. Combined nivolumab and Ipilimumab in Melanoma Metastatic to the Brain. N Engl J Med. (2018) 379:722-30. doi: 10.1056/NEJMoa1805453

26. Long GV, Atkinson V, Cebon JS, Jameson MB, Fitzharris BM, McNeil $\mathrm{CM}$, et al. Standard-dose pembrolizumab in combination with reduceddose ipilimumab for patients with advanced melanoma (KEYNOTE029): an open-label, phase lb trial. Lancet Oncol. (2017) 18:120210. doi: 10.1016/S1470-2045(17)30428-X

27. Goldberg SB, Gettinger SN, Mahajan A, Chiang AC, Herbst RS, Sznol $\mathrm{M}$, et al. Pembrolizumab for patients with melanoma or non-smallcell lung cancer and untreated brain metastases: early analysis of a non-randomised, open-label, phase 2 trial. Lancet Oncol. (2016) 17:97683. doi: 10.1016/S1470-2045(16)30053-5

28. Yamazaki $\mathrm{N}$, Takenouchi $\mathrm{T}$, Fujimoto $\mathrm{M}$, Ihn $\mathrm{H}$, Uchi $\mathrm{H}$, Inozume $\mathrm{T}$, et al. Phase $1 \mathrm{~b}$ study of pembrolizumab (MK-(3475) antiPD-1 monoclonal antibody) in Japanese patients with advanced melanoma (KEYNOTE-041). Cancer Chemother Pharmacol. (2017) 79:651-60. doi: 10.1007/s00280-016-3237-x

29. Robert C, Schachter J, Long GV, Arance A, Grob JJ, Mortier L, et al. Pembrolizumab versus Ipilimumab in Advanced Melanoma. $N$ Engl J Med. (2015) 372:2521-32. doi: 10.1056/NEJMoa1503093

30. Ribas A, Puzanov I, Dummer R, Schadendorf D, Hamid O, Robert C, et al. Pembrolizumab versus investigator-choice chemotherapy for ipilimumabrefractory melanoma (KEYNOTE-002): a randomised, controlled, phase 2 trial. Lancet Oncol. (2015) 16:908-18. doi: 10.1016/S1470-2045(15)00083-2

31. Hamid O, Robert C, Daud A, Hodi FS, Hwu WJ, Kefford R, et al. Safety and tumor responses with lambrolizumab (anti-PD-1) in melanoma. $N$ Engl J Med. (2013) 369:134-44. doi: 10.1056/NEJMoa1305133

32. Robert C, Ribas A, Wolchok JD, Hodi FS, Hamid O, Kefford $\mathrm{R}$, et al. Anti-programmed-death-receptor-1 treatment with pembrolizumab in ipilimumab-refractory advanced melanoma: a randomised dose-comparison cohort of a phase 1 trial. Lancet. (2014) 384:1109-17. doi: 10.1016/S0140-6736(14)60958-2

33. Ribas A, Hamid O, Daud A, Hodi FS, Wolchok JD, Kefford R, et al. Association of pembrolizumab with tumor response and survival among patients with advanced melanoma. JAMA. (2016) 315:1600-9. doi: 10.1001/jama.2016.4059

34. Topalian SL, Sznol M, McDermott DF, Kluger HM, Carvajal RD, Sharfman $\mathrm{WH}$, et al. Survival, durable tumor remission, and long-term safety in patients with advanced melanoma receiving nivolumab. J Clin Oncol. (2014) 32:102030. doi: 10.1200/JCO.2013.53.0105

35. Weber JS, Kudchadkar RR, Yu B, Gallenstein D, Horak CE, Inzunza $\mathrm{HD}$, et al. Safety, efficacy, and biomarkers of nivolumab with vaccine in ipilimumab-refractory or -naive melanoma. J Clin Oncol. (2013) 31:43118. doi: 10.1200/JCO.2013.51.4802

36. Weber JS, D'Angelo SP, Minor D, Hodi FS, Gutzmer R, Neyns B, et al Nivolumab versus chemotherapy in patients with advanced melanoma 
who progressed after anti-CTLA-4 treatment (CheckMate 037): a randomised, controlled, open-label, phase 3 trial. Lancet Oncol. (2015) 16:375-84. doi: 10.1016/S1470-2045(15)70076-8

37. Robert C, Long GV, Brady B, Dutriaux C, Maio M, Mortier L, et al. Nivolumab in previously untreated melanoma without BRAF mutation. $N$ Engl J Med. (2015) 372:320-30. doi: 10.1056/NEJMoa1412082

38. Yamazaki N, Kiyohara Y, Uhara H, Uehara J, Fujimoto M, Takenouchi T, et al. Efficacy and safety of nivolumab in Japanese patients with previously untreated advanced melanoma: a phase II study. Cancer Sci. (2017) 108:122330. doi: $10.1111 /$ cas. 13241

39. Margolin K, Ernstoff MS, Hamid O, Lawrence D, McDermott D, Puzanov I, et al. Ipilimumab in patients with melanoma and brain metastases: an open-label, phase 2 trial. Lancet Oncol. (2012) 13:45965. doi: 10.1016/S1470-2045(12)70090-6

40. Chesney J, Puzanov I, Collichio F, Singh P, Milhem MM, Glaspy J, et al. Randomized, open-label phase II study evaluating the efficacy and safety of talimogene laherparepvec in combination with ipilimumab versus ipilimumab alone in patients with advanced, unresectable melanoma. J Clin Oncol. (2018) 36:1658-67. doi: 10.1200/JCO.2017.73.7379

41. Ascierto PA, Del Vecchio M, Robert C, Mackiewicz A, Chiarion-Sileni $\mathrm{V}$, Arance $\mathrm{A}$, et al. Ipilimumab $10 \mathrm{mg} / \mathrm{kg}$ versus ipilimumab $3 \mathrm{mg} / \mathrm{kg}$ in patients with unresectable or metastatic melanoma: a randomised, double-blind, multicentre, phase 3 trial. Lancet Oncol. (2017) 18:61122. doi: 10.1016/S1470-2045(17)30231-0

42. Weber J, Hamid O, Amin A, O’Day S, Masson E, Goldberg SM, et al. Randomized phase I pharmacokinetic study of Ipilimumab with or without one of two different chemotherapy regimens in patients with untreated advanced melanoma. Cancer Immun. (2013) 13:7.

43. Hodi FS, O’Day SJ, McDermott DF, Weber RW, Sosman JA, Haanen JB, et al. Improved survival with ipilimumab in patients with metastatic melanoma. $N$ Engl J Med. (2010) 363:711-23. doi: 10.1056/NEJMoa1003466

44. Yamazaki N, Kiyohara Y, Uhara H, Fukushima S, Uchi H, Shibagaki N, et al. Phase II study of ipilimumab monotherapy in Japanese patients with advanced melanoma. Cancer Chemother Pharmacol. (2015) 76:9971004. doi: 10.1007/s00280-015-2873-x

45. Wolchok JD, Neyns B, Linette G, Negrier S, Lutzky J, Thomas L, et al. Ipilimumab monotherapy in patients with pretreated advanced melanoma: a randomised, double-blind, multicentre, phase 2, dose-ranging study. Lancet Oncol. (2010) 11:155-64. doi: 10.1016/S1470-2045(09)70334-1

46. Weber J, Thompson JA, Hamid O, Minor D, Amin A, Ron I, et al. A randomized, double-blind, placebo-controlled, phase II study comparing the tolerability and efficacy of ipilimumab administered with or without prophylactic budesonide in patients with unresectable stage III or IV melanoma. Clin Cancer Res. (2009) 15:5591-8. doi: 10.1158/1078-0432.CCR-09-1024

47. Zimmer L, Eigentler TK, Kiecker F, Simon J, Utikal J, Mohr P, et al. Open-label, multicenter, single-arm phase II DeCOG-study of ipilimumab in pretreated patients with different subtypes of metastatic melanoma. J Transl Med. (2015) 13:351. doi: 10.1186/s12967-015-0716-5

48. Haag GM, Zoernig I, Hassel JC, Halama N, Dick J, Lang N, et al. Phase II trial of ipilimumab in melanoma patients with preexisting humoural immune response to NY-ESO-1. Eur J Cancer. (2018) 90:1229. doi: 10.1016/j.ejca.2017.12.001

49. Hodi FS, Lee S, McDermott DF, Rao UN, Butterfield LH, Tarhini AA, et al. Ipilimumab plus sargramostim vs ipilimumab alone for treatment of metastatic melanoma: a randomized clinical trial. JAMA. (2014) 312:174453. doi: 10.1001/jama.2014.13943

50. Weber JS, O'Day S, Urba W, Powderly J, Nichol G, Yellin M, et al. Phase I/II study of ipilimumab for patients with metastatic melanoma. J Clin Oncol. (2008) 26:5950-6. doi: 10.1200/JCO.2008.16.1927

51. Hersh EM, O'Day SJ, Powderly J, Khan KD, Pavlick AC, Cranmer LD, et al. A phase II multicenter study of ipilimumab with or without dacarbazine in chemotherapy-naive patients with advanced melanoma. Invest New Drugs. (2011) 29:489-98. doi: 10.1007/s10637-009-9376-8

52. Downey SG, Klapper JA, Smith FO, Yang JC, Sherry RM, Royal RE, et al. Prognostic factors related to clinical response in patients with metastatic melanoma treated by CTL-associated antigen-4 blockade. Clin Cancer Res. (2007) 13(22 Pt 1):6681-8. doi: 10.1158/1078-0432.CCR-07-0187
53. Maker AV, Yang JC, Sherry RM, Topalian SL, Kammula US, Royal $\mathrm{RE}$, et al. Intrapatient dose escalation of anti-CTLA-4 antibody in patients with metastatic melanoma. J Immunother. (2006) 29:455-63. doi: 10.1097/01.cji.0000208259.73167.58

54. Callahan MK, Kluger H, Postow MA, Segal NH, Lesokhin A, Atkins MB, et al. Nivolumab plus ipilimumab in patients with advanced melanoma: updated survival, response, and safety data in a phase I dose-escalation study. J Clin Oncol. (2018) 36:391-8. doi: 10.1200/JCO.2017.72.2850

55. Hodi FS, Chiarion-Sileni V, Gonzalez R, Grob JJ, Rutkowski P, Cowey CL, et al. Nivolumab plus ipilimumab or nivolumab alone versus ipilimumab alone in advanced melanoma (CheckMate 067): 4-year outcomes of a multicentre, randomised, phase 3 trial. Lancet Oncol. (2018) 19:148092. doi: 10.1016/S1470-2045(18)30700-9

56. Hodi FS, Chesney J, Pavlick AC, Robert C, Grossmann KF, McDermott DF, et al. Combined nivolumab and ipilimumab versus ipilimumab alone in patients with advanced melanoma: 2-year overall survival outcomes in a multicentre, randomised, controlled, phase 2 trial. Lancet Oncol. (2016) 17:1558-68. doi: 10.1016/S1470-2045(16)30366-7

57. Kluger HM, Chiang V, Mahajan A, Zito CR, Sznol M, Tran T, et al. LongTerm Survival of Patients With Melanoma With Active Brain Metastases Treated With Pembrolizumab on a Phase II Trial. J Clin Oncol. (2019) 37:52-60. doi: 10.1200/JCO.18.00204

58. Schachter J, Ribas A, Long GV, Arance A, Grob JJ, Mortier L, et al. Pembrolizumab versus ipilimumab for advanced melanoma: final overall survival results of a multicentre, randomised, openlabel phase 3 study (KEYNOTE-006). Lancet. (2017) 390:185362. doi: 10.1016/S0140-6736(17)31601-X

59. Hamid O, Puzanov I, Dummer R, Schachter J, Daud A, Schadendorf $\mathrm{D}$, et al. Final analysis of a randomised trial comparing pembrolizumab versus investigator-choice chemotherapy for ipilimumab-refractory advanced melanoma. Eur J Cancer. (2017) 86:37-45. doi: 10.1016/j.ejca.2017.07.022

60. Weber J, Gibney G, Kudchadkar R, Yu B, Cheng P, Martinez AJ, et al. Phase I/II study of metastatic melanoma patients treated with nivolumab who had progressed after ipilimumab. Cancer Immunol Res. (2016) 4:34553. doi: 10.1158/2326-6066.CIR-15-0193

61. Hamid O, Schmidt H, Nissan A, Ridolfi L, Aamdal S, Hansson J, et al. A prospective phase II trial exploring the association between tumor microenvironment biomarkers and clinical activity of ipilimumab in advanced melanoma. J Transl Med. (2011) 9:204. doi: 10.1186/1479-5876-9-204

62. O’Day SJ, Maio M, Chiarion-Sileni V, Gajewski TF, Pehamberger H, Bondarenko IN, et al. Efficacy and safety of ipilimumab monotherapy in patients with pretreated advanced melanoma: a multicenter single-arm phase II study. Ann Oncol. (2010) 21:1712-7. doi: 10.1093/annonc/mdq013

63. El Osta B, Hu F, Sadek R, Chintalapally R, Tang SC. Not all immunecheckpoint inhibitors are created equal: Meta-analysis and systematic review of immune-related adverse events in cancer trials. Crit Rev Oncol Hematol. (2017) 119:1-12. doi: 10.1016/j.critrevonc.2017.09.002

64. Boutros C, Tarhini A, Routier E, Lambotte O, Ladurie FL, Carbonnel F, et al. Safety profiles of anti-CTLA-4 and anti-PD-1 antibodies alone and in combination. Nat Rev Clin Oncol. (2016) 13:473-86. doi: 10.1038/nrclinonc.2016.58

65. Zhang B, Wu Q, Zhou YL, Guo X, Ge J, Fu J. Immune-related adverse events from combination immunotherapy in cancer patients: a comprehensive meta-analysis of randomized controlled trials. Int Immunopharmacol. (2018) 63:292-8. doi: 10.1016/j.intimp.2018.08.014

66. Costa R, Carneiro BA, Agulnik M, Rademaker AW, Pai SG, Villaflor VM, et al. Toxicity profile of approved anti-PD-1 monoclonal antibodies in solid tumors: a systematic review and meta-analysis of randomized clinical trials. Oncotarget. (2017) 8:8910-20. doi: 10.18632/oncotarget.13315

67. Komaki Y, Komaki F, Yamada A, Micic D, Ido A, Sakuraba A. Meta-analysis of the risk of immune-related adverse events with anticytotoxic t-lymphocyteassociated antigen 4 and antiprogrammed death 1 therapies. Clin Pharmacol Ther. (2018) 103:318-31. doi: 10.1002/cpt.633

68. Bertrand A, Kostine M, Barnetche T, Truchetet ME, Schaeverbeke $\mathrm{T}$. Immune related adverse events associated with anti-CTLA-4 antibodies: systematic review and meta-analysis. BMC Med. (2015) 13:211. doi: 10.1186/s12916-015-0455-8 
69. Wang PF, Chen Y, Song SY, Wang TJ, Ji WJ, Li SW, et al. Immune-related adverse events associated with anti-PD-1/PD-L1 treatment for malignancies: a meta-analysis. Front Pharmacol. (2017) 8:730. doi: 10.3389/fphar.2017.00730

70. de Filette J, Andreescu CE, Cools F, Bravenboer B, Velkeniers B. A Systematic review and meta-analysis of endocrine-related adverse events associated with immune checkpoint inhibitors. Horm Metab Res. (2019) 51:14556. doi: 10.1055/a-0843-3366

71. Baxi S, Yang A, Gennarelli RL, Khan N, Wang Z, Boyce L, et al. Immunerelated adverse events for anti-PD-1 and anti-PD-L1 drugs: systematic review and meta-analysis. BMJ. (2018) 360:k793. doi: 10.1136/bmj.k793

72. Lu J, Yang J, Liang $Y$, Meng $H$, Zhao J, Zhang $X$. Incidence of immune checkpoint inhibitor-associated diabetes: a meta-analysis of randomized controlled studies. Front. Pharmacol. (2019) 10:1453. doi: 10.3389/fphar.2019.01453

73. Mellati M, Eaton KD, Brooks-Worrell BM, Hagopian WA, Martins R, Palmer JP, et al. Anti-PD-1 and Anti-PDL-1 monoclonal antibodies causing type 1 diabetes. Diabetes Care. (2015) 38:e137-8. doi: 10.2337/ dc15-0889

74. Usui Y, Udagawa H, Matsumoto S, Imai K, Ohashi K, Ishibashi M, et al. Association of serum anti-GAD antibody and HLA haplotypes with type 1 diabetes mellitus triggered by nivolumab in patients with non-small cell lung cancer. J Thorac Oncol. (2017) 12:e41-3. doi: 10.1016/j.jtho.2016. 12.015

75. Way J, Drakaki A, Drexler A, Freeby M. Anti-PD-L1 therapy and the onset of diabetes mellitus with positive pancreatic autoantibodies. BMJ Case Rep. (2017) 2017. doi: 10.1136/bcr-2017-220415
76. Dechartres A, Trinquart L, Boutron I, Ravaud P. Influence of trial sample size on treatment effect estimates: meta-epidemiological study. BMJ. (2013) 346:f2304. doi: 10.1136/bmj.f2304

77. Kumar V, Chaudhary N, Garg M, Floudas CS, Soni P, Chandra AB. Current diagnosis and management of Immune Related Adverse Events (irAEs) induced by immune checkpoint inhibitor therapy. Front Pharmacol. (2017) 8:49. doi: 10.3389/fphar.2017.00049

78. Chen TW, Razak AR, Bedard PL, Siu LL, Hansen AR. A systematic review of immune-related adverse event reporting in clinical trials of immune checkpoint inhibitors. Ann Oncol. (2015) 26:1824-9. doi: 10.1093/annonc/mdv182

79. Teramukai S, Matsuyama Y, Mizuno S, Sakamoto J. Individual patient-level and study-level meta-analysis for investigating modifiers of treatment effect. Jpn J Clin Oncol. (2004) 34:717-21. doi: 10.1093/jjco/hyh138

Conflict of Interest: The authors declare that the research was conducted in the absence of any commercial or financial relationships that could be construed as a potential conflict of interest.

Copyright (C) 2020 Almutairi, McBride, Slack, Erstad and Abraham. This is an openaccess article distributed under the terms of the Creative Commons Attribution License (CC BY). The use, distribution or reproduction in other forums is permitted, provided the original author(s) and the copyright owner(s) are credited and that the original publication in this journal is cited, in accordance with accepted academic practice. No use, distribution or reproduction is permitted which does not comply with these terms. 